\title{
Decoupled Hydrodynamic Models and Their Outdoor Identification for an Unmanned Inland Cargo Vessel with Embedded Fully Rotatable Thrusters
}

\author{
Gerben Peeters $^{1,2, *} \mathbb{D}$, Senne Van Baelen ${ }^{1}\left(\mathbb{D}\right.$, Gökay Yayla $^{1}(\mathbb{D})$, Tim Catoor ${ }^{1}$, \\ Muhammad Raheel Afzal ${ }^{1}{ }^{1}$, Christos Christofakis ${ }^{1}$, Robrecht Louw ${ }^{1}\left(\mathbb{D}\right.$, , Yogang Singh ${ }^{1}{ }^{(1)}$, \\ Maarten Vanierschot ${ }^{1}$, , René Boonen ${ }^{1}$ and Peter Slaets ${ }^{1, *(\mathbb{D})}$ \\ 1 KU Leuven, Mechanical Engineering Technology, Andreas Vesaliusstraat 13, 3000 Leuven, Belgium; \\ senne.vanbaelen@kuleuven.be (S.V.B.); gokay.yayla@kuleuven.be (G.Y.); tim.catoor@kuleuven.be (T.C.); \\ raheel.afzal@kuleuven.be (M.R.A.); christos.christofakis@kuleuven.be (C.C); \\ robrecht.louw@kuleuven.be (R.L.); yogang.singh@kuleuven.be (Y.S.); \\ maarten.vanierschot@kuleuven.be (M.V); rene.boonen@kuleuven.be (R.B.) \\ 2 Research Foundation-Flanders (FWO), Egmontstraat 5, 1000 Brussels, Belgium \\ * Correspondence: gerben.peeters@kuleuven.be (G.P.); peter.slaets@kuleuven.be (P.S.)
}

Received: 19 October 2020; Accepted: 5 November 2020; Published: 7 November 2020

\begin{abstract}
Expanding the automation level of the freshly introduced fleet of self-propelled Watertruck ${ }^{+}$barges, which house fully-rotatable embedded thrusters, might increase their ability to compete with their less sustainable but dominating road-based alternatives. Hydrodynamic motion models, which reveal the manoeuvring capabilities of these barges, can serve as inputs for many pieces of this automation puzzle. No identified motion models or hydrodynamic data seem to be publicly available for the hull design and the novel actuation system configuration of these barges. Therefore, this study offers: (i) decoupled motion model structures for these barges for surge, sway, and yaw, with a focus on the thruster and damping models; (ii) two identification procedures to determine these motion models; (iii) all the experimental data, generated outdoors with a scale model barge to identify (i) based on (ii). In addition, the identified surge models were compared with both computational and empirical data. These comparisons offer more physical insights into the identified model structures and can aid in the model selection for which the desired complexity and accuracy evidently depend on their envisaged application. Finally, this methodology need not be limited to the vessel and actuation types utilised by us.
\end{abstract}

Keywords: manoeuvring; hydrodynamic; model; identification; vessel; rotatable; thrusters; surge; sway; yaw

\section{Introduction}

The European Watertruck ${ }^{+}$project [1] aims to revivify the inland waterway transport sector by introducing a novel modular fleet of push vessels and active or passive barges of CEMT hull types I and II [2], which enables the decoupling between sailing and transshipment time. With the purpose of studying the automation potential of this new fleet, Peeters et al. [3] constructed a scale model of a self-propelled Watertruck ${ }^{+}$barge in conjunction with a complementary inland shore control centre [4] to monitor or control this barge. Several links in this envisaged automation chain can benefit from a hydrodynamic model which describes the motion of this vessel. For example, these models can serve as plant models to verify or tune control algorithms, or as control models to construct model predictive controllers [5], which can also be used for collision avoidance algorithms [6,7]. Furthermore, Kotzé et al. [8] and Peeters et al. [4] suggested that these motion models could be applied to construct 
dynamic proximity zones surrounding the vessel hull, which could be shared with other ambient vessels or could trigger internal control or monitoring events.

The literature handling these hydrodynamic models appears to hold four main approaches: (i) Transfer functions can produce response models, e.g., relating yaw-rates with rudder changes $[9,10]$; (ii) Abkowitz [11] introduced a third-order Taylor expansion model to mathematically describe the forces acting on a vessel around its equilibrium state [12]; (iii) artificial neural networks can model the ship manoeuvring motion [13]; and (iv) physically oriented modular models—such as the modular mathematical model [14] and the robot-like vectorial model [15,16] — can offer more physical insights in the vessel dynamics. This study uses the robot-like vectorial model given that it leverages system properties such as symmetry, skew-symmetry, and positiveness of matrices [17], which align with the envisaged future utilisation of the models in this work.

No public hydrodynamic data appear to be available for the CEMT type I-II hulls, and little for inland vessels in general [18]. In addition, the novel self-propelled barges have a non-conventional propulsion system consisting of two fully nested and 360-degree-steerable actuators: a steering grid thruster in the bow and a four-channel thruster in the stern, for which little or no models or data seem to be available too. This propulsion configuration enables a high level of manoeuvrability, crucial for inland vessels which need to navigate independently [19]. Therefore, this study investigates decoupled hydrodynamic motion models for surge, sway, and yaw. This decoupling offers insights in the vessel manoeuvrability on the one hand, and enables a focused investigation of the thruster models on the other hand. More precisely, this study aimed to use the scale model vessel to:

(i) Compare different model structures for the damping forces and non-conventional thrusters, with an emphasis on the potential speed-dependent propulsive forces of these thrusters.

(ii) Provide two identification methods to determine the coefficients of these or other manoeuvring models: one method uses the instantaneous force balance, and the other one uses the integrated system dynamics by solving the differential equations of motion.

(iii) Benchmark the results of the surge models with data from both a computational fluid dynamics (CFD) study [20] and an empirical approach [21].

(iv) Provide all the experimental data of this study in S1 Experimental Data (see Supplementary Materials), together with the details of the utilised data logging and processing approaches.

The achievement of these aims provides more understanding of the manoeuvrability of these vessels and the potential hydrodynamic models to predict such movements in surge, sway, and yaw. In addition, these decoupled models present a stepping stone for subsequent coupled models in the future, and the supplemental experimental data unlock the possibility for other researchers to investigate this novel Watertruck ${ }^{+}$fleet. This paper continues as follows: Section 2 details the design of the scale model vessel, named the Cogge. Thereupon, Section 3 lists the methods used to achieve the results of Section 4. Finally, Section 5 provides an overarching discussion of these results, and Section 6 concludes this work.

\section{Materials}

Section 2.1 handles the geometrical similarities between the Cogge and the real-size Watertruck ${ }^{+}$ barges, and Section 2.2 details their unconventional actuation configurations. Afterwards, Section 2.3 lists the most relevant onboard components used during the outdoor experiments with the Cogge.

\subsection{Geometry of the Cogge}

Figure 1 a depicts a push boat pushing four coupled Watertruck ${ }^{+}$barges and Figure $1 \mathrm{~b}$ shows

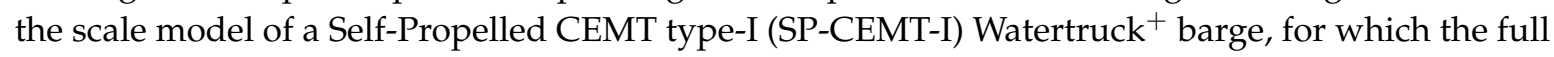
design details can be found in [3]. Table 1 presents the geometrical similarity—by using a scale factor of $\lambda=8$-between the Cogge and the SP-CEMT-I vessels. The resultant size of the Cogge makes it physically impossible to board this vessel, turning it into an unmanned surface vessel. 


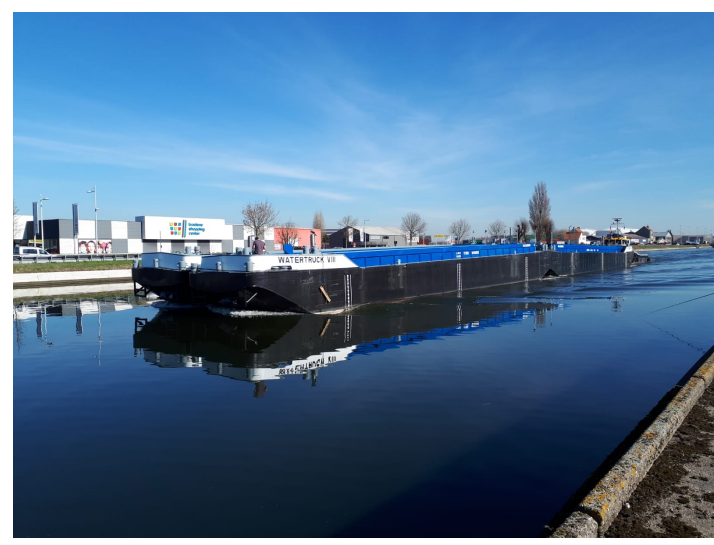

(a)

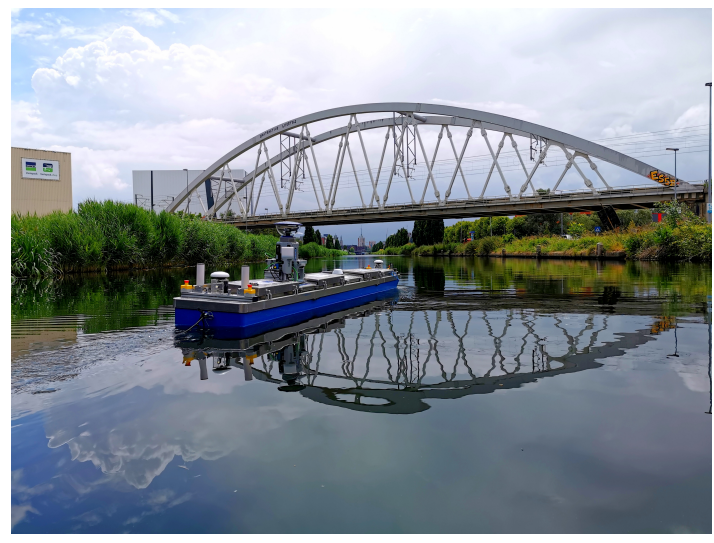

(b)

Figure 1. The Watertruck ${ }^{+}$concept: (a) four full-sized barges pushed by a push boat [1], reproduced from [4] with permission from MDPI, 2020; (b) the KU Leuven scale model of a self-propelled barge named Cogge [3].

Table 1. Designed geometry of the Cogge and the self-propelled Watertruck ${ }^{+}$barges. $^{-}$

\begin{tabular}{lrrrl}
\hline Parameter & Symbol & CEMT-I & Cogge & Units \\
\hline Scale & $\lambda$ & 1 & $8^{-1}$ & {$[-]$} \\
Length & $\mathrm{L}$ & 38.50 & 4.81 & {$[\mathrm{~m}]$} \\
Beam & $\mathrm{B}$ & 5.05 & 0.63 & {$[\mathrm{~m}]$} \\
Draft $_{\text {full }}$ & $\mathrm{T}_{\text {full }}$ & 2.80 & 0.35 & {$[\mathrm{~m}]$} \\
Block coefficient & $C_{B}$ & 0.95 & 0.95 & {$[-]$} \\
\hline
\end{tabular}

\subsection{0-Degree-Rotatable Thrusters}

Figure 2a outlines a bottom view of the hull geometry of the Cogge, demonstrating the water inlets for both thruster systems: on the left hand side for the four-channel stern thruster, and on the right hand side for the steering-grid bow thruster. Both thrusters draw in water via these inlets and exhaust a water stream according to the orientation of their steering mechanism. To illustrate the working principles of these steering mechanisms, Figure 2b,c shows longitudinal cross-sections through the centres of the four-channel and steering-grid thrusters, respectively. Figure 2d,e similarly presents the internal angle-conventions $-\alpha_{c}^{i}$ and $\alpha_{g}^{i}$ - of these thrusters and the working principle of their steering mechanisms, by means of an abstract functional drawing. The steering mechanism of the four channel thruster consists of half a sphere with an opening of approximately $85^{\circ}$ which can be rotated $360^{\circ}$. However, the water flow can only exit the vessel through one or two of its four channels, introducing a non-linear mapping between $\alpha_{c}^{i}$ and the resultant orientation of its thrust force. Note that the two longitudinal channels, see Figure $2 b$, have a small downwards angle, whereas the two-not shown-transversal channels have no such bending. The steering grid thruster can also exhaust its water flow in a full $360^{\circ}$ range. Figure $2 \mathrm{c}$ indicates that this systems bends the flow by approximately $180^{\circ}$ in the zx-plane before it exits the system. The grid itself has a small angle $\beta$ to orient the flow underneath the hull.

The bollard pull forces of both thrusters can be found in Figure A1, and more design details together with their main discrepancies with conventional actuation systems in [22]. Since the bow of the Cogge houses the steering-grid and the stern the four-channel thruster, $\alpha_{b}^{i}$ and $\alpha_{s}^{i}$ will denote their internal angles in this study, and $n_{b}$ and $n_{s}$ their propeller speeds. Furthermore, in [3] a flow straightener was placed inside the stern-oriented exhaust channel of the stern thruster to increase the straight line stability of the Cogge. This self made straightener was not installed during the bollard pull tests from Figure A1. 


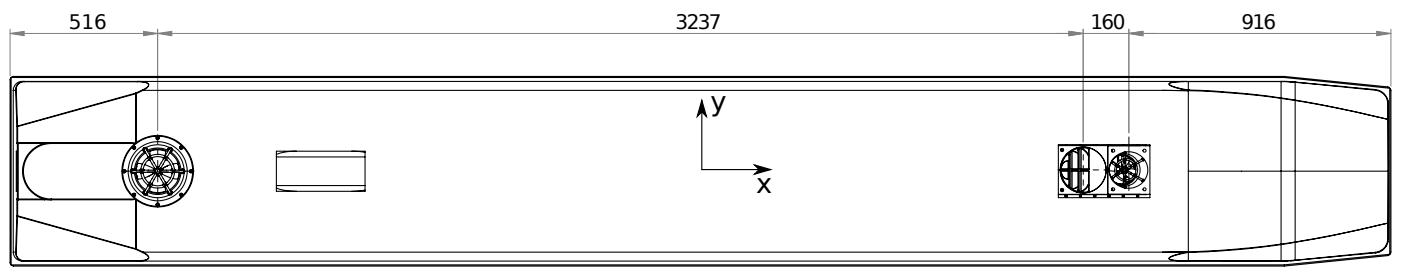

(a)

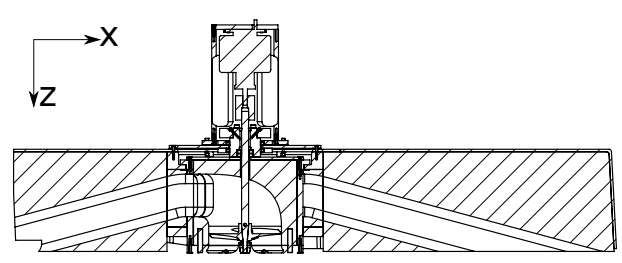

(b)

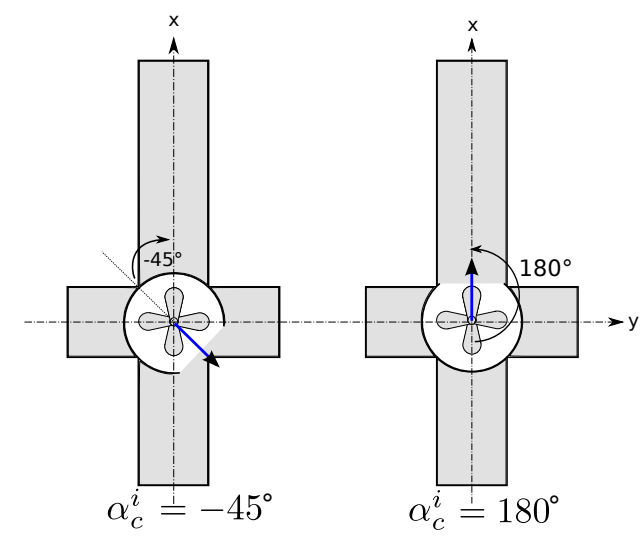

(d)

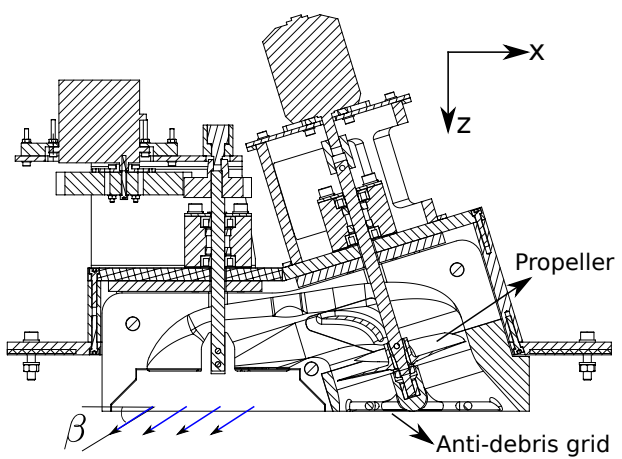

(c)

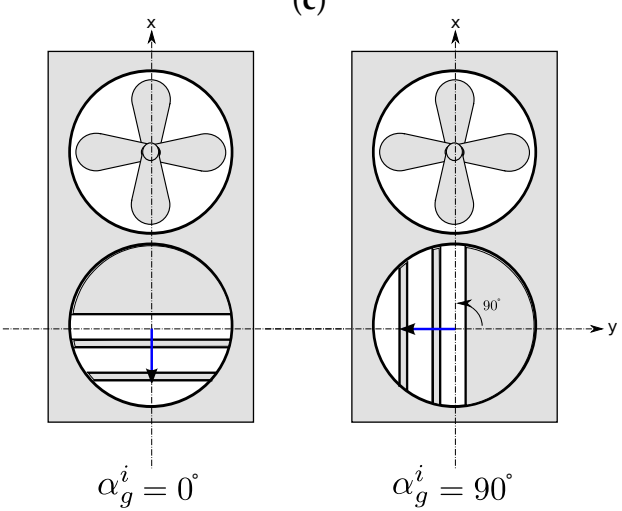

(e)

Figure 2. Actuation system design: (a) positions of both thrusters; (b) longitudinal cross-section of the four-channel thruster at the stern; (c) longitudinal cross-section of the steering-grid at the bow; (d) angle convention four-channel thruster with $\alpha_{c}^{i}=-45^{\circ}$ and $=180^{\circ}$; and (e) angle convention steering-grid with $\alpha_{g}^{i}=0^{\circ}$ and $=90^{\circ}$. Figures $(\mathbf{b}-\mathbf{e})$ were adapted or reproduced from [22] with permission from MDPI, 2020.

\subsection{Main Onboard Components}

Figure 3 summarises the main onboard components and their communication links, for which more details can be found in [3,4]. In summary, a programmable logic controller (PLC) controls the internal thruster angles and rotational speeds of the actuators based on their desired values, which it receives from an industrial computer (I-PC)—or remote controller or Web interface. During the experiments, this I-PC ran the Mission Oriented Operating Suite (MOOS) middleware [23] and received data from the GNSS and IMU sensors. An AsteRx-U Marine Global Navigation Satellite System (GNSS) receiver with two PolaNt* MC antennas [24] was pulse per second (PPS) synchronised with an Ekinox-2E Inertial Measurement Unit (IMU) [25]. The GNSS sensor received real-time kinematic (RTK) corrections via the Flemish Positioning Service, which currently has 45 RTK base stations installed [26]. With these RTK corrections, this GNSS sensor claims to have a horizontal accuracy-depending on the distance to the nearest base station-of $0.6 \mathrm{~cm}+0.5 \mathrm{ppm}$, expressed in twice the distance root mean square. The main GNSS antenna, placed at the stern, expressed the position data and was separated by approximately $4.44 \mathrm{~m}$ from the auxiliary antenna, at the bow, which enabled accurate pitch and yaw data [3]. 
The IMU sensor ran a real-time extended Kalman filter at $200 \mathrm{~Hz}$, where it received corrective steps from the GNSS unit at $5 \mathrm{~Hz}$. A calibration procedure- to find the position and orientation differences between the IMU and GNSS sensors-was conducted using the IMU software; more details and a benchmark of this procedure can be found in [27]. In this study, both the GNSS sensor and the IMU internally logged-PPS synchronised-data at $50 \mathrm{~Hz}$. The GNSS sensor also sent the Coordinated Universal Time (UTC) to the I-PC, where the MOOS middleware logged these data together with the desired and measured actuation system states which were requested at $20 \mathrm{~Hz}$. Accordingly, the UTC stamps provided the connection for the post-processing synchronisation of all the relevant data; see Section 3.2.2.

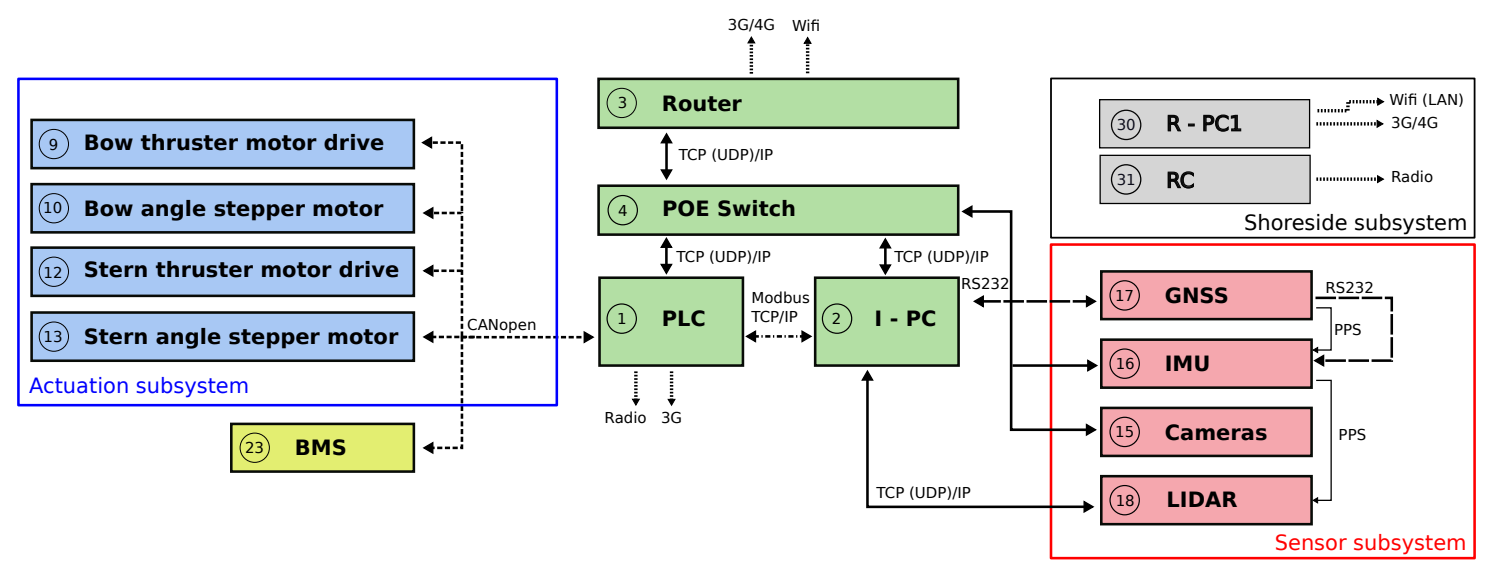

Figure 3. Onboard components and their communication links reproduced from [4] with permission from MDPI, 2020.

\section{Methods}

Section 3.1 starts with deriving the decoupled equations of motion for surge, sway, and yaw, which will be used throughout this study. Section 3.2 discusses the design of the experiments to generate data in order to identify these models, for which Section 3.3 offers two identification methods. Finally, Section 3.4 provides an overview of the used approaches to compare the results of these identification procedures.

\subsection{Decoupled Hydrodynamic Motion Models}

\subsubsection{Modelling Assumptions}

Equation (1) describes the equations of motion for a vessel in calm water, and thus with frequency-independent coefficients, according to the robot-like vectorial model of $[15,17]$ :

$$
\underbrace{M_{R B} \dot{v}+C_{R B}(v) v}_{\text {rigid-body forces }}+\underbrace{M_{A} \dot{v}+C_{A}(v) v+D(v) v}_{\text {hydrodynamicforces }}+\underbrace{g(\eta) \eta+g_{o}}_{\text {hydrostaticforces }}=\tau_{\text {external }}
$$

Here $v=[u, v, w, p, q, r]^{\top}$ denotes the generalised velocity vector expressed in a body-fixed reference frame with origin, $O_{b}$, and $\eta=[x, y, z, \phi, \theta, \psi]^{\top}$ the generalised position and orientation vector expressed in an inertial reference frame with origin, $O_{i} ;$ Figure 4 illustrates their convention throughout this study. The matrix $M_{R B}$ represents the rigid-body mass matrix, $C_{R B}$ the rigid-body Coriolis and centripetal matrix, $\boldsymbol{M}_{A}$ the added mass matrix, $C_{A}$ the Coriolis and centripetal matrix due to $\boldsymbol{M}_{A}, \boldsymbol{D}(\boldsymbol{v})$ the damping matrix, $g(\eta)$ the gravitational/buoyancy vector, $g_{0}$ the ballast control vector, and finally, $\tau_{\text {external }}$ accumulates all the external forces acting on the vessel. 


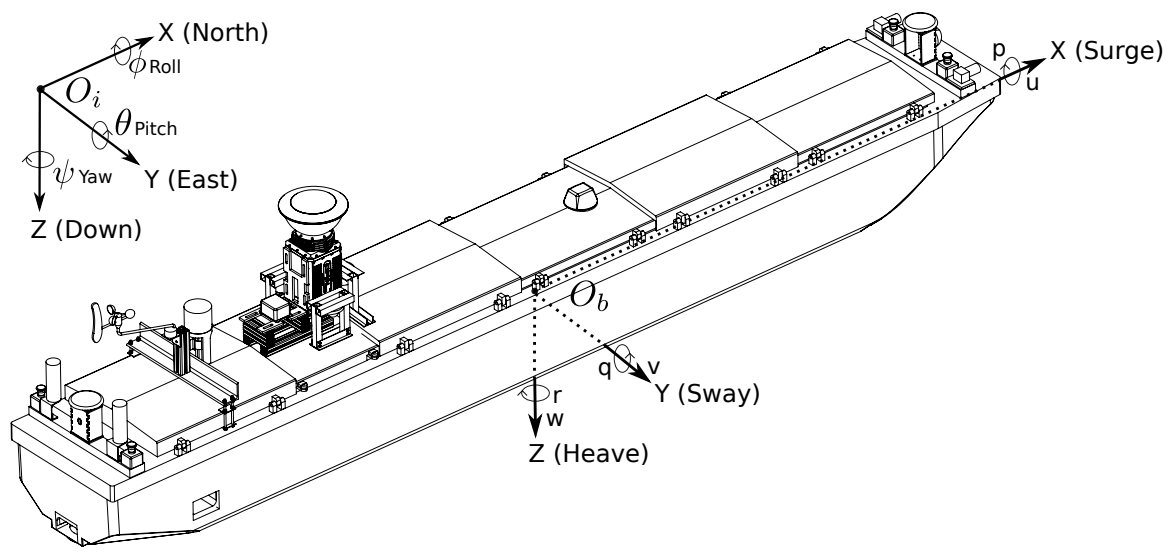

Figure 4. Inertial $\left(O_{i}\right)$ and body-fixed $\left(O_{b}\right)$ reference frames.

The following assumptions were made to model the decoupled hydrodynamic equations of motion for the barge in this study:

i. A local north-east-down reference frame approximates an inertial reference frame to describe the position and orientation of the vessel navigating over short distances; see Figure 4.

ii. $\mathrm{O}_{b}$ is positioned at the centre of gravity and its axes align with the principal directions of the barge. Furthermore, given the rather prism-shaped geometry of the vessel and its block coefficient of 0.95 , the horizontal positions of the centre of gravity, buoyancy, and floatation are assumed to coincide in the middle of the vessel in both length and width.

iii. The experimental design of this study aimed at solely studying, and thus exciting, the horizontal motions of the vessel, i.e., neglecting pitch, roll, and heave motions, meaning: $\eta=[x, y, \psi]^{\top}$ and $v=[u, v, r]^{\top}$. Furthermore, the remaining motions in the water plane were investigated separately for each degree of freedom. Subsequently, no Coriolis, centripetal, buoyancy, or ballast forces were modelled.

iv. Only thruster related external forces were explicitly modelled, i.e., $\tau_{\text {external }}=\boldsymbol{\tau}_{\text {thrust }}$, and their dynamic behaviour was neglected. Do note that for the surge and sway models a bias term was added to the external forces, which was intended to implicitly capture any constant wind forces; see Section 3.3.

The accumulation of these four main assumptions results in the following set of equations which represent the decoupled hydrodynamic motions for the barge in surge, sway, and yaw motion:

$$
\left(\boldsymbol{M}+\boldsymbol{M}_{\boldsymbol{A}}\right) \dot{\boldsymbol{v}}+\boldsymbol{D}(\boldsymbol{v}) \boldsymbol{v}=\boldsymbol{\tau}_{\text {thrust }}
$$

Accordingly, three hydrodynamic phenomena need to be modelled and identified: the added mass, damping, and actuation forces, which are respectively detailed in Sections 3.1.2-3.1.4.

\subsubsection{Added Mass Models}

In the manoeuvring theory framework [17], the added masses [28] can be modelled by a constant term. Several approaches exist to determine these terms in the absence of well-conditioned towing tank data: (i) numerical approaches (strip theory [29] or panel methods [30]) which are often based on (ii) theoretical values [29,31] for common shapes, (iii) empirical methods [32,33], and (iv) less-conditioned outdoor experiments. Liu et al. [18] successfully implemented the empirical methods of Zhou et al. [32] and Clarke et al. [33] on two scale model reference inland vessels for the Yangtze River. Therefore, this study applied the method of Zhou et al. [32] for the transversal and rotational added masses, and the method of Clarke et al. [33] for the longitudinal added mass, $X_{\dot{u}}$, which equates to:

$$
3 \% \times M \leq X_{\dot{u}} \leq 6 \% \times M,
$$


where $M$ denotes the mass of the vessel. Given that our vessel has a higher $C_{B}$ than the reference vessels used to derive (3) [33], the upper limit of (3) was be selected for $X_{\dot{u}}$. Additionally, Zhou et al. [32] suggested that the transversal, $Y_{\dot{v}}$, and rotational, $N_{\dot{r}}$ added masses can be approximated based on the main ship dimensions, listed in Table 1, by the following two relations:

$$
\begin{gathered}
Y_{\dot{v}}=M\left[0.882-0.54 C_{B}\left(1-1.6 \frac{T}{B}\right)-0.156\left(1-0.673 C_{B}\right) \frac{L}{B}\right. \\
\left.+0.826 \frac{T}{B} \frac{L}{B}\left(1-0.678 \frac{T}{B}\right)-0.638 C_{B} \frac{T}{B} \frac{L}{B}\left(1-0.669 \frac{T}{B}\right)\right], \\
N_{\dot{r}}=M j_{z}^{2}, \quad j_{z}=\frac{L}{100}\left[33-76.85 C_{B}\left(1-0.784 C_{B}\right)+3.43 \frac{L}{B}\left(1-0.63 C_{B}\right)\right] .
\end{gathered}
$$

\subsubsection{Damping Models}

The arising damping forces have many known contributors, e.g., potential damping (due to wave forming), skin friction, vortex shedding, and lifting forces (both due to linear circulation of the water around the hull and due to cross-flow drag). Different modelling assumptions exist to model this complex array of contributors [17,34-36]. Given that: (i.) it is not convenient to independently identify all these contributors, and (ii.) this study investigates the decoupled planar motions of the barge, the complexity of the one degree of freedom damping models was chosen to be cubic, quadratic, or linear, respectively represented by the following three equations:

$$
\begin{gathered}
D(u)=X_{u u u} u^{3}+X_{u u} u^{2}+X_{u} u, \quad D(v)=Y_{v v v} v^{3}+Y_{v v} v^{2}+Y_{v} v, \quad D(r)=N_{r r r} r^{3}+N_{r r} r^{2}+N_{r} r \\
D(u)=X_{u u} u^{2}+X_{u} u, \quad D(v)=Y_{v v} v^{2}+Y_{v} v, \quad D(r)=N_{r r} r^{2}+N_{r} r \\
D(u)=X_{u} u, \quad D(v)=Y_{v} v, \quad D(r)=N_{r} r
\end{gathered}
$$

\subsubsection{Propulsion System Models}

In the spectrum of the more conventional propulsion systems, tunnel thrusters [37-39] and azimuth thrusters [40-42] might appear to hold the largest similarities with the non-conventional thrusters of this study [22]. The modelling literature for these tunnel and azimuth thrusters does remain scarce and often uses the open-water propeller characteristics model of [43] as the starting point. Furthermore, the present actuation system might look similar to a water jet system, but it was not designed to perform at the conventional higher speed ranges for these systems [44,45], nor does the overall inlet and outlet design seem to be optimal for such purposes [46]. Do note that some water jet models [47] use the findings of [43] as a modelling starting point, for consistency with the existing hydrodynamic literature. Subsequently, the present work will also start with the first order approximation of the lift forces induced by the propeller blades [43]. Accordingly, the theoretical thrust force, $T_{t h}$, depends quadratically on the shaft speed, $n$, and bi-linearly on $n$ and the axial inflow speed $V_{A}$ :

$$
T_{t h}\left(n, V_{A}\right)=T_{n n} n^{2}+T_{n_{V_{A}}} n V_{A}
$$

Introducing the non-dimensional thrust coefficient $K_{T}$ and advance ratio $J$ :

$$
K_{T}=\frac{T_{t h}}{\rho n^{2} D_{p}^{4}}, \quad J=\frac{V_{A}}{n D_{p}},
$$

where $\rho$ represents the water density and $D_{p}$ the propeller diameter, in conjunction with a linear approximation of $K_{T}$ :

$$
K_{T}=K_{T_{0}}+K_{T_{J}} J
$$


gives rise to their connection with (9):

$$
T_{t h}=K_{T_{0}} \rho D_{p}^{4} n^{2}+K_{T_{J}} \rho D_{p}^{3} n V_{A}
$$

where

$$
T_{n n}=K_{T_{0}} \rho D_{p}^{4}, T_{n V_{A}}=K_{T_{J}} \rho D_{p}^{3} .
$$

Appending a propeller behind a ship hull changes (9) due to propeller-hull and propeller-wake interactions which can be modelled by the thrust deduction coefficient, $t$, and the wake fraction, $w$, respectively:

$$
V_{A}=(1-w) u, \quad T=(1-t) T_{t h},
$$

with $T$ the actually available thrust force. Combining (9) and (14) results in:

$$
T(n, u, t, w)=(1-t)\left[T_{n n} n^{2}+T_{n_{V_{A}}} n(1-w) u\right],
$$

for a conventionally-positioned propeller on a vessel with forward speed. Given (i) the present 360-degrees-rotatable actuators (see Section 2.2), (ii) their angle-dependent bollard thrust forces (see Section 2.2 and Figure A1), and (iii) the manoeuvrability of the vessel, $t$ could be made angle-dependent, and $w$ velocity-dependent, as follows:

$$
\left.T\left(n, v, \alpha^{i}, t, w\right)=1-t\left(\alpha^{i}\right)\right]\left[T_{n n} n^{2}+(1-w(v)) T_{n v} n v\right] .
$$

In [22], the authors demonstrated that $T(v=0)=\left[1-t\left(\alpha^{i}\right)\right] T_{n n} n^{2}$ can serve as a robust theoretical thrust force model for the speed-independent part of (16), by studying the data from Figure A1. Note that $t$ and $w$ denote thrust differences between fully open-water tests (only a propeller) and tests with a propeller attached to a hull. Therefore, the static parts of $t\left(\alpha^{i}\right)$ and $w(v)$ cannot be explicitly identified in this study, but they will be implicitly incorporated in the thrust coefficients. Nevertheless, their respective angle- and speed-dependency can be investigated.

For the experiments with decoupled vessel motions, i.e., $v=[u, 0,0]^{\top},[0, v, 0]^{\top}$, or $[0,0, r]^{\top}$, three thruster angles suffice, $\alpha^{i} \in[-90,0,90]^{\circ}$ (see Section 3.2.1), and assuming vessel and actuator symmetry around the zx-plane narrows these angles down to $\alpha^{i} \in[0,90=-90]^{\circ}$. Furthermore, the water speed at the inlets of the propellers will be assumed to be purely longitudinal or transversal, where for the rotational case the inlet transversal speed at the bow or stern thruster depends on their longitudinal distance $L_{b / s}^{x}$ from the centre of gravity $\left(v_{\text {inlet }}=L_{b / s}^{x}\right)$. In addition, given the axisymmetric water inlet at the bottom of the ship hull and the position of the propeller shaft which stands orthogonal to the calm water plane, it will be further assumed that the differences in wake effects for a longitudinal or transversal inlet flow will be negligible. This assumption means, $w(v)$ can be modelled by a single term, $w(v)=w_{v} v$, where $v$ will be assumed to be either pure $u$ or $v$. Combining these assumptions allows for a further simplification of the thruster models:

$$
\begin{aligned}
T\left(0^{o}\right)=T\left(n, v=u, \alpha^{i}=0^{o}\right) & =\left(1-t\left(0^{o}\right)\right)\left[T_{n n} n^{2}+\left(1-w_{v}\right) T_{n v} n u\right] \\
& =T_{n n}^{0} n^{2}+T_{n v}^{0} n u \\
T\left(90^{\circ}\right)=-T\left(-90^{\circ}\right)=T\left(n, v=v, \alpha^{i}\right. & \left.=90^{\circ}\right)=\left(1-t\left(90^{\circ}\right)\right)\left[T_{n n} n^{2}+\left(1-w_{v}\right) T_{n v} n v\right] \\
& =T_{n n}^{90} n^{2}+T_{n v}^{90} n v
\end{aligned}
$$

The thruster model of (17) and (18) will be coined the conventional thrust model in this study. Two other models will be explored too: (19) the bollard thrust model and (20) the direct speed loss thrust model, in order to offer more insights into the potential speed dependency of the thrust forces:

$$
T(0)=T_{n n}^{0} n^{2}, \quad T(90)=T_{n n}^{90} n^{2},
$$




$$
T(0)=T_{n n}^{0} n^{2}+T_{n n v}^{0} n^{2} u, \quad T(90)=T_{n n}^{90} n^{2}+T_{n n v}^{90} n^{2} v
$$

\subsubsection{Selected Decoupled Hydrodynamic Motion Models}

Table 2 lists the selected combinations of the aforementioned model structures for the damping and thruster forces. The resultant model structures, $\mathcal{M}\left(\boldsymbol{\theta}_{d}^{x}\right)$, are named by the vector of their unknown parameters, $\boldsymbol{\theta}_{\boldsymbol{d}}^{\boldsymbol{x}}$, for each degree of freedom, $d \in[u, v, r]$, and a combination of thruster and damping models, $x \in[a, b, c, d, e, f, g, h, i]$. In order to fully crystallise this convention, (21) expresses the full model of $\mathcal{M}\left(\boldsymbol{\theta}_{\boldsymbol{u}}^{a}\right)$, (22) of $\mathcal{M}\left(\boldsymbol{\theta}_{\boldsymbol{v}}^{e}\right)$, and (23) of $\mathcal{M}\left(\boldsymbol{\theta}_{\boldsymbol{r}}^{\boldsymbol{i}}\right)$, where the superscripts ${ }^{b}$ and ${ }^{s}$ for the thruster parameters denote the bow and stern thruster respectively.

$$
\begin{gathered}
\mathcal{M}\left(\boldsymbol{\theta}_{u}^{a}\right)=\left(M+X_{\dot{u}}\right) \dot{u}+X_{u u u} u^{3}+X_{u u} u^{2}+X_{u} u=T_{n n}^{0, b} n_{b}^{2}+T_{n v}^{0, b} n_{b} u+T_{n n}^{0, s} n_{s}^{2}+T_{n v}^{0, s} n_{s} u \\
\mathcal{M}\left(\boldsymbol{\theta}_{v}^{e}\right)=\left(M+Y_{\dot{v}}\right) \dot{v}+Y_{v v} v^{2}+Y_{v} v=T_{n n}^{90, b} n_{b}^{2}+T_{n n}^{90, s} n_{s}^{2} \\
\mathcal{M}\left(\boldsymbol{\theta}_{r}^{i}\right)=\left(I_{z}+N_{\dot{r}}\right) \dot{r}+N_{r} r=L_{b}^{x}\left(T_{n n}^{90, b} n_{b}^{2}+T_{n n v}^{90, b} n_{b}^{2} L_{b}^{x} r\right)+L_{s}^{x}\left(T_{n n}^{-90, s} n_{s}^{2}+T_{n n v}^{-90, s} n_{s}^{2} L_{s}^{x} r\right)
\end{gathered}
$$

Table 2. The selected model structures of this study.

\begin{tabular}{lrrr}
\hline Model & Added Mass & Damping & Propulsion \\
\hline $\mathcal{M}\left(\boldsymbol{\theta}_{\boldsymbol{u}}^{\boldsymbol{a}}\right), \mathcal{M}\left(\boldsymbol{\theta}_{\boldsymbol{v}}^{\boldsymbol{a}}\right), \mathcal{M}\left(\boldsymbol{\theta}_{r}^{\boldsymbol{a}}\right)$ & Constant, see (3)-(5) & Cubic, see (6) & Conventional Thrust, see (17) \\
$\mathcal{M}\left(\boldsymbol{\theta}_{\boldsymbol{u}}^{\boldsymbol{b}}\right), \mathcal{M}\left(\boldsymbol{\theta}_{\boldsymbol{v}}^{\boldsymbol{b}}\right), \mathcal{M}\left(\boldsymbol{\theta}_{r}^{\boldsymbol{b}}\right)$ & Constant, see (3)-(5) & Quadratic, see (7) & Conventional Thrust, see (18) \\
$\mathcal{M}\left(\boldsymbol{\theta}_{\boldsymbol{u}}^{\boldsymbol{c}}\right), \mathcal{M}\left(\boldsymbol{\theta}_{\boldsymbol{v}}^{\boldsymbol{c}}\right), \mathcal{M}\left(\boldsymbol{\theta}_{r}^{\boldsymbol{c}}\right)$ & Constant, see (3)-(5) & Linear, see (8) & Conventional Thrust, see (18) \\
$\mathcal{M}\left(\boldsymbol{\theta}_{\boldsymbol{u}}^{\boldsymbol{d}}\right), \mathcal{M}\left(\boldsymbol{\theta}_{\boldsymbol{v}}^{\boldsymbol{d}}\right), \mathcal{M}\left(\boldsymbol{\theta}_{r}^{\boldsymbol{d}}\right)$ & Constant, see (3)-(5) & Cubic, see (6) & Bollard Thrust, see (19) \\
$\mathcal{M}\left(\boldsymbol{\theta}_{\boldsymbol{u}}^{\boldsymbol{e}}\right), \mathcal{M}\left(\boldsymbol{\theta}_{\boldsymbol{v}}^{\boldsymbol{e}}\right), \mathcal{M}\left(\boldsymbol{\theta}_{r}^{\boldsymbol{e}}\right)$ & Constant, see (3)-(5) & Quadratic, see (7) & Bollard Thrust, see (19) \\
$\mathcal{M}\left(\boldsymbol{\theta}_{\boldsymbol{u}}^{f}\right), \mathcal{M}\left(\boldsymbol{\theta}_{\boldsymbol{v}}^{f}\right), \mathcal{M}\left(\boldsymbol{\theta}_{r}^{f}\right)$ & Constant, see (3)-(5) & Linear, see (8) & Bollard Thrust, see (19) \\
$\mathcal{M}\left(\boldsymbol{\theta}_{\boldsymbol{u}}^{\boldsymbol{g}}\right), \mathcal{M}\left(\boldsymbol{\theta}_{\boldsymbol{v}}^{\boldsymbol{g}}\right), \mathcal{M}\left(\boldsymbol{\theta}_{r}^{\boldsymbol{g}}\right)$ & Constant, see (3)-(5) & Cubic, see (6) & Direct Speed Loss, see (20) \\
$\mathcal{M}\left(\boldsymbol{\theta}_{\boldsymbol{u}}^{\boldsymbol{h}}\right), \mathcal{M}\left(\boldsymbol{\theta}_{\boldsymbol{v}}^{\boldsymbol{h}}\right), \mathcal{M}\left(\boldsymbol{\theta}_{r}^{\boldsymbol{h}}\right)$ & Constant, see (3)-(5) & Quadratic, see (7) & Direct Speed Loss, see (20) \\
$\mathcal{M}\left(\boldsymbol{\theta}_{\boldsymbol{u}}^{\boldsymbol{i}}\right), \mathcal{M}\left(\boldsymbol{\theta}_{\boldsymbol{v}}^{\boldsymbol{i}}\right), \mathcal{M}\left(\boldsymbol{\theta}_{r}^{\boldsymbol{i}}\right)$ & Constant, see (3)-(5) & Linear, see (8) & Direct Speed Loss, see (20) \\
\hline
\end{tabular}

\subsection{Experimental Design}

\subsubsection{Decoupled Surge, Sway, and Yaw Motion Experiments}

In the comparative review of identification papers on ship manoeuvring models in [48], it can be noted that most studies use standard manoeuvres as inputs. In this study, the decoupled motions of surge, sway, and yaw need to be excited independently over a variety of speeds, in order to generate data for the models of Section 3.1.5. Table 3 shows that the actuation system of the Cogge enables three submodes to generate surge motion data: (i) bow thruster only, named "Bow", (ii) stern thruster only, named "Stern", and (iii) both thrusters simultaneously, named "Dual". In addition, the Cogge can sway to the $1^{\text {"Left" }}$ " or to the "Right", not unlike the crabbing test manoeuvres [49]. Finally, a counter thrust test as suggested by [50] can be performed clockwise or counter-clockwise, named "CW" or "CCW", to induce the yaw motion data.

For each of the aforementioned submodes, three desired time-dependent propeller speed missions, $n_{b / s}^{d}(t)$, were requested from the thrusters named "Ramp," "Stairs," and "Step" in Table 3, and each mission was conducted twice. The first batch of missions, denoted by " $m_{1}$ ", were conducted in calm weather conditions, whereas a slight 1-2 Beaufort wind picked up during the second batch, " $m_{2}$ ", which can also be seen in Section 4.3.1. For the surge missions, both thrusters had an equal internal angle, $\alpha_{b / s}^{i}=0^{\circ}$, for the sway missions both angles were oriented sideways, $\alpha_{b / s}^{i}=90^{\circ}$ or $=-90^{\circ}$, and for the yaw missions both thrusters had opposite sideway angles, $\alpha_{b}^{i}=-\alpha_{s}^{i}=90^{\circ}$ or $=-90^{\circ}$. Figure 5 illustrates the three different desired and measured, $n_{b / s}^{m}(t)$, time-dependent propeller speed profiles. The requested and measured rotational speeds for all missions can be found in the Supplementary Materials S1 Experimental Data. For the sway and yaw motions, the $n_{b / s}^{d}$ were manually determined during the experiments and set to $n_{b}^{d}=2000 \mathrm{rpm}$ and $n_{s}^{d}=500 \mathrm{rpm}$ for all 
their submodes. This speed ratio gave good visual results-by watching the vessel motion from inside a supply vessel—for both sway and yaw, but it introduces a small discrepancy: since a no-yaw requirement needs equal but counteracting moments of the thrusters, and a no-sway requirement needs equal but counteracting forces of these thrusters. Given the different lever arms of both thrusters, see Figure 2a, it might be assumed that one cannot achieve both situations, i.e., no yaw during sway and vice versa, with the same propeller speed ratio, although this depends on the potential speed-dependency of the thrust forces which was unknown during the experiments.

Table 3. Overview of all experiments.

\begin{tabular}{lccccccc}
\hline & \multicolumn{3}{c}{ Surge } & \multicolumn{2}{c}{ Sway } & \multicolumn{2}{c}{ Yaw } \\
\hline $\boldsymbol{n}_{\boldsymbol{b} / \boldsymbol{s}}^{\boldsymbol{d}}(\boldsymbol{t})$ & Bow & Stern & Dual & Left & Right & CW & CCW \\
\hline Ramp & $m_{1}, m_{2}$ & $m_{1}, m_{2}$ & $m_{1}, m_{2}$ & $m_{1}, m_{2}$ & $m_{1}, m_{2}$ & $m_{1}, m_{2}$ & $m_{1}, m_{2}$ \\
Stairs & $m_{1}, m_{2}$ & $m_{1}, m_{2}$ & $m_{1}, m_{2}$ & $m_{1}, m_{2}$ & $m_{1}, m_{2}$ & $m_{1}, m_{2}$ & $m_{1}, m_{2}$ \\
Step & $m_{1}, m_{2}$ & $m_{1}, m_{2}$ & $m_{1}, m_{2}$ & $m_{1}, m_{2}$ & $m_{1}, m_{2}$ & $m_{1}, m_{2}$ & $m_{1}, m_{2}$ \\
\hline
\end{tabular}

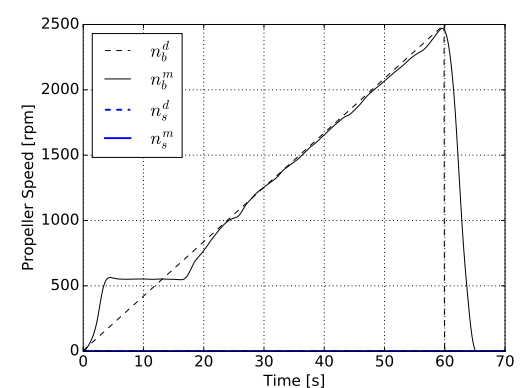

(a) bow-ramp- $m_{1}$

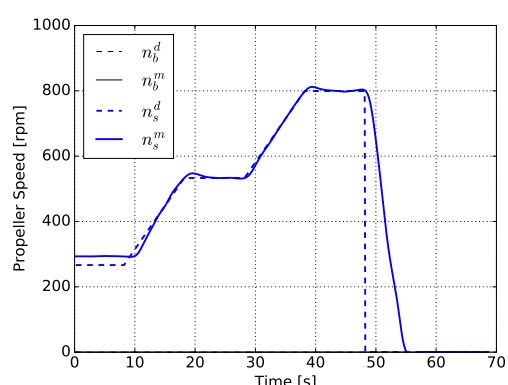

(b) stern-stairs- $m_{1}$

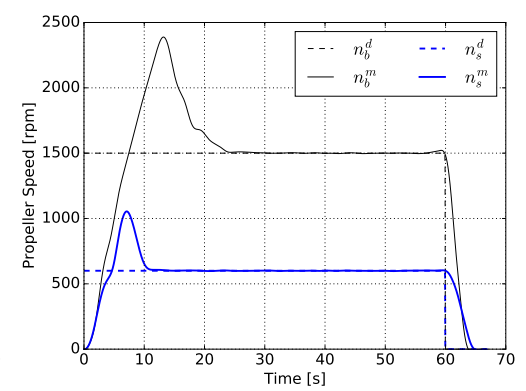

(c) dual-step- $m_{1}$

Figure 5. Time-dependent speed profiles, desired $\left(n_{b / s}^{d}\right)$ and measured $\left(n_{b / s}^{m}\right)$ values: (a) ramp profile for a bow thruster mission, (b) stair profile for a stern thruster mission, and (c) a step profile for a dual thrusters mission.

\subsubsection{Data Post Processing}

Due to the different data logging locations and frequencies (see Section 2.3), the relative time vector of the MOOS database needed to be converted to UTC stamps, and the MOOS data needed to be merged with the other sensor data. Consequently, the following manipulations were done to the MOOS data using the Pandas package [51] in Python version 3 [52]: (i) the time vector was converted to a UTC time vector, (ii) the data were clipped to their relevant mission time range, (iii) the offsets in relative time and position were removed, (iv) the variables were linearly interpolated, given that MOOS allows for asynchronous data logging, and (v) the resultant data were upsampled to $50 \mathrm{~Hz}$. Afterwards, these data were merged with the IMU and GNSS data based on the UTC time vector. The potential time delays between a measurement and its log were assumed negligible and irrelevant compared to the studied vessel dynamics.

The Supplementary Materials S1 Experimental Data holds the results of these manipulations for the variables used in this study in a file per mission, structured according to the taxonomy of Table 3. As a result, each mission file contains: (i) a relative time vector, (ii) the position data of the main GNSS mushroom, which were calculated in the MOOS in real-time using the driver from [53], (iii) the desired and measured $\alpha_{b / s}^{i}$ and $n_{b / s}$ from the MOOS, (iv) the roll, pitch, and yaw angles from the IMU, (v) the yaw-rate from the IMU, (vi) the northern and eastern speeds from the IMU, and (vii) the surge and sway accelerations from the IMU. Note that the IMU publishes these accelerations in a horizontal ship reference frame where the gravity vector has been taken into account. This horizontal frame aligns with the assumptions made in Section 3.1.1. In combination with the decoupled design of the experiments, and thus by neglecting other motions, one can assume these accelerations to be equal to those at the centre of gravity for the surge and sway experiments. 
Based on the logged northern and eastern speeds, the course over ground was calculated which allowed the determination of the drift vector. Thereupon, the surge and sway speeds were calculated based on the total speed and drift vectors. In addition, the yaw-accelerations were calculated using the gradient function from Numpy [54]. Finally, all data-to have similar distortions on each variablewere filtered forward and backward — to avoid phase distortions — using a low pass Butterworth filter of order 2 with desired cutoff frequency of $0.3 \mathrm{~Hz}$ and Nyquist frequency of $25 \mathrm{~Hz}$, by using the Scipy package [55]. Note that these calculations and filters were not added or performed on the Supplementary Material S1 Experimental Data, in order to provide the original as-measured data sets.

\subsection{Identification Methods}

The offline identification of hydrodynamic vessel models can be roughly divided into frequency and time domain methods $[48,56,57]$. This study used two different time domain approaches of which the usage need not be restricted to the models suggested in this study. The first method uses the instantaneous force balance of the dynamic equations, given that all inputs and outputs were measured during the experiments; see Section 3.3.1. The second approach integrates the system dynamics by solving its differential equations; see Section 3.3.2. For each model structure $x \in[a, \cdots, i]$ and motion $d \in[u, v, r]$, both methods called the bounded nonlinear least squares function of Virtanen et al. [55], which uses a thrust-region reflective method based on Branch et al. [58] to minimise a cost function, $E_{d}^{x}\left(\theta_{d}^{x}\right)$, for $\mathrm{k}$ sets of data by altering the values of their parameter vector $\boldsymbol{\theta}_{d}^{x}$ :

$$
E_{d}^{x}\left(\theta_{d}^{x}\right)=\sum_{i=1}^{i=k} E_{d_{i}}^{x}\left(\theta_{d}^{x}\right)
$$

To each translational training mission, i.e., $d \in[u, v]$, a bias term $\beta_{d_{i}}$ was added as an external force, which was intended to capture the static parts of the wind forces. Accordingly, the vector $\beta_{d}=\left[\beta_{d_{i}}, \ldots, \beta_{d_{k}}\right]$ was added to the $\theta_{d}^{x}$ vectors of the surge and sway cases. In other words, for these two cases, the $k$ training missions share the unknown parameter vector $\theta_{d}^{x}$ but each mission has an individual bias term $\beta_{d_{i}}$. To facilitate the notation of the cost functions in Sections 3.3.1 and 3.3.2, let the matrices $y_{d_{i}}=\left[\dot{d}_{i}, d_{i}^{3}, d_{i}^{2}, d_{i}\right]$ accumulate all the speeds and their quadratic and cubic values, and the accelerations for each $d \in[u, v, r]$. Similarly, let $x_{d_{i}}=\left[n_{s}^{m}, n_{b}^{m}, \alpha_{s}^{m}, \alpha_{b}^{m}\right]$ capture all the measured thruster states. For $x_{u_{i}}$ and $x_{v_{i}}$, the outputs $u_{i}$ and $v_{i}$ should be added too, given that some thruster models depend on these speeds. Similarly, $x_{r_{i}}$ needs to be appended with the calculated transversal speeds at the thrusters: $v_{b, i}$ and $v_{s, i}$. Consequently, each parameter vector $\theta_{d}^{x}$ can be similarly divided into two parts which accumulate the parameters relevant for $y_{d_{i}}$ and $x_{d_{i}}$ respectively: $\theta_{d}^{x_{y}}$ and $\theta_{d}^{x_{x}}$. Finally, all "left" and "CCW" missions were transformed to "right" and "CW" missions by changing the signs of the measurements.

\subsubsection{Force Balance Method (FBM)}

Using the knowledge from (2), i.e., the inertial and damping forces should equal the thrust forces, and by adding the individual potential wind bias term for each translation mission, the following three equations show the cost functions for $k$ training sets for the surge, sway, and yaw methods respectively using the FBM approach:

$$
\begin{gathered}
E_{u}^{x}\left(\boldsymbol{\theta}_{u}^{x}, \beta_{u}\right)=\sum_{i=1}^{i=k} E_{u_{i}}^{x}\left(\boldsymbol{\theta}_{u}^{x}, \beta_{u_{i}}\right)=\sum_{i=1}^{i=k}\left(\boldsymbol{\theta}_{u}^{x_{y}} \boldsymbol{y}_{u_{i}}-\boldsymbol{\theta}_{u}^{x_{x}} \boldsymbol{x}_{\boldsymbol{u}_{i}}-\beta_{u_{i}}\right)^{2} \\
E_{v}^{x}\left(\boldsymbol{\theta}_{\boldsymbol{v}}^{x}, \boldsymbol{\beta}_{\boldsymbol{v}}\right)=\sum_{i=1}^{i=k} E_{v_{i}}^{x}\left(\boldsymbol{\theta}_{v}^{x}, \beta_{v_{i}}\right)=\sum_{i=1}^{i=k}\left(\boldsymbol{\theta}_{v}^{x_{y}} \boldsymbol{y}_{v_{i}}-\boldsymbol{\theta}_{v}^{x_{x}} \boldsymbol{x}_{v_{i}}-\beta_{v_{i}}\right)^{2} \\
E_{r}^{x}\left(\boldsymbol{\theta}_{r}^{x}\right)=\sum_{i=1}^{i=k} E_{r_{i}}^{x}\left(\boldsymbol{\theta}_{r}^{x}\right)=\sum_{i=1}^{i=k}\left(\boldsymbol{\theta}_{r}^{x_{y}} \boldsymbol{y}_{r_{i}}-\boldsymbol{\theta}_{r}^{x_{x}} \boldsymbol{x}_{\boldsymbol{r}_{i}}\right)^{2}
\end{gathered}
$$




\subsubsection{Differential Equation Method (DEM)}

In order to integrate the differential equations of motion, only the measured $\alpha_{b / s}^{i}$ and $n_{b / s}$ are needed in combination with the initial vessel speed, which provides the start condition. These integrations were done using the "odeint" function from the SciPy python package [55] which calls the "lsode" solver from the FORTRAN library "odepack" [59]. To further clarify this approach, Equation (28) expresses such a differential equation for $\mathcal{M}\left(\boldsymbol{\theta}_{v}^{e}\right)$ of (22):

$$
\dot{v}=\frac{T_{n n}^{b, 90} n_{b}^{2}+T_{n n}^{s, 90} n_{s}^{2}-Y_{v v} v^{2}-Y_{v} v}{M+Y_{\dot{v}}}
$$

Integrating (28) for each time period of $20 \mathrm{~ms}$, where the associated propeller speed and angle measurements are updated accordingly, generates a vector, $\hat{v}$, with the predicted sway speeds for that mission. The vectors $\hat{u}$ and $\hat{r}$ can be calculated analogously. The DEM cost functions aim to minimise the difference between these predicted speeds and the measured speeds by changing the values of $\boldsymbol{\theta}_{\boldsymbol{d}}^{x}$ and $\beta_{d}$, as respectively shown for surge, sway, and yaw speeds:

$$
\begin{gathered}
E_{u}^{x}\left(\boldsymbol{\theta}_{u}^{x}, \boldsymbol{\beta}_{u}\right)=\sum_{i=1}^{i=k} E_{u_{i}}^{x}\left(\boldsymbol{\theta}_{\boldsymbol{u}}^{x}, \beta_{u_{i}}\right)=\sum_{i=1}^{i=k}\left(\boldsymbol{u}_{i}-\hat{\boldsymbol{u}}_{\boldsymbol{i}}\left(t, \boldsymbol{x}_{u_{i}}, \boldsymbol{\theta}_{\boldsymbol{u}}^{x}, \beta_{u_{i}}\right)\right)^{2} \\
E_{v}^{x}\left(\boldsymbol{\theta}_{\boldsymbol{v}}^{x}, \boldsymbol{\beta}_{\boldsymbol{v}}\right)=\sum_{i=1}^{i=k} E_{v_{i}}^{x}\left(\boldsymbol{\theta}_{\boldsymbol{v}}^{x}, \beta_{v_{i}}\right)=\sum_{i=1}^{i=k}\left(\boldsymbol{v}_{\boldsymbol{i}}-\hat{\boldsymbol{v}}_{\boldsymbol{i}}\left(t, \boldsymbol{x}_{v_{i}}, \boldsymbol{\theta}_{v}^{x}, \beta_{v_{i}}\right)\right)^{2} \\
E_{r}^{x}\left(\boldsymbol{\theta}_{r}^{x}\right)=\sum_{i=1}^{i=k} E_{r_{i}}^{x}\left(\boldsymbol{\theta}_{r}^{x}\right)=\sum_{i=1}^{i=k}\left(\boldsymbol{r}_{\boldsymbol{i}}-\hat{\boldsymbol{r}}_{\boldsymbol{i}}\left(t, \boldsymbol{x}_{\boldsymbol{r}_{i}}, \boldsymbol{\theta}_{r}^{x}\right)\right)^{2}
\end{gathered}
$$

Thus, this DEM method uses an implementation of the prediction error method philosophy [60]. In comparison to methods using the known predictor equation, e.g., [61], which only predicts one step ahead based on the previous measurement, the DEM method can predict the speed profile for the whole time range based on $\theta_{d}^{x}$ and $\beta_{d}$. Furthermore, the equations of motion need no linearisation, which often is the case (e.g., $[62,63])$, and no direct accelerations need to be used. The extended Kalman filter of the IMU does use these accelerations to calculate its speeds, so they are implicitly nested in the cost functions.

\subsection{Model Structure Comparison and Selection}

Section 3.4.1 offers the comparison potential for the surge identification results with external data sets from a CFD study and an empirical approach. Afterwards, Section 3.4.2 briefly lists the comparison possibilities solely using the data of this study.

\subsubsection{Comparisons with External Data}

Peeters et al. [20] applied CFD to investigate the surge resistance of the Cogge. Accordingly, they simulated its hydrodynamic behaviour at five surge speeds, $u \in[0.2,0.4,0.6,0.8,1.0] \frac{\mathrm{m}}{\mathrm{s}}$, with the OpenFOAM [64] software, using the volume of fluid approach [65] and a $k-\omega$ turbulence model [66]. In order to verify their modelling approach, they first conducted the same methodology on a scale model KVLCC2 hull for which towing tank data were present [67]. For this vessel, six speed simulations in the range of $u=0.944$ to $1.283 \frac{\mathrm{m}}{\mathrm{s}}$ resulted in a maximal error of $1.55 \%$ compared with the towing tank data. To examine their spatial grid convergence, they used only two grids which resulted in a grid convergence index (GCI) estimation [68] for their coarser grid of around 7\%, which depends on the applied safety factor and assumed grid convergence. This GCI suggests an error band around the asymptotic value of the simulations, in which the current solution lies. The calculations for the Cogge were done on a finer grid refinement compared to this coarser grid for the KVLCC2 hull. Hence, perhaps it might be assumed that its GCI would lie in the same order of magnitude. 
Figure 6a shows the relative pressure field of the water surface around the Cogge, which was longitudinally cut in the middle, had a draft of $T_{C F D}=0.23 \mathrm{~m}$, and had a simulated speed of $u=1.0 \frac{\mathrm{m}}{\mathrm{s}}$. For the same configurations, Figure $6 \mathrm{~b}$ depicts the water speed and streamlines for a longitudinal section of water at the middle of the vessel, i.e., at the top of Figure 6a. Figure $6 \mathrm{~b}$ demonstrates that, during the initial design phase, the steering-grid thruster at the bow protruded the vessel hull. The physical Cogge has no such protrusion, since its thrusters are fully embedded. In addition, these simulations were done with a total vessel height of $0.35 \mathrm{~m}$, whereas the final hull had a height of approximately $0.43 \mathrm{~m}$, if sensor extensions were neglected. Bearing these assumptions and discrepancies in mind, the CFD calculations resulted in the following second order approximation of the surge damping forces:

$$
D_{C F D}(u)=22.12 u^{2}+0.10 u
$$

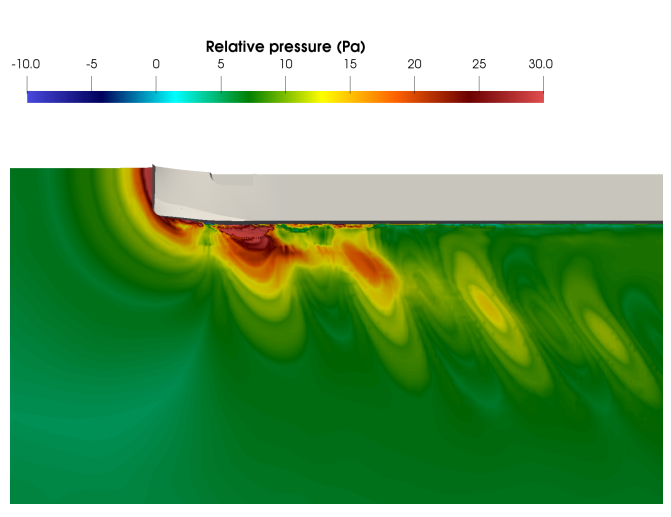

(a)

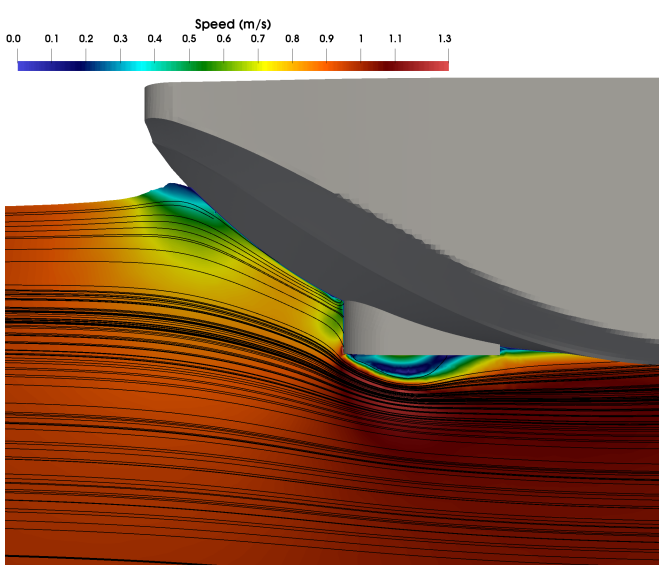

(b)

Figure 6. CFD results with $u=1.0 \frac{\mathrm{m}}{\mathrm{s}}$, reproduced from [20] with permission from IEEE, 2020: (a) relative pressure field on the water's surface, and (b) speed and streamlines for a longitudinal section of the water at the bow.

Kristensen et al. [21] extensively reported on an empirical approach based on the ITTC-1957 method [69] to estimate the damping forces of a vessel. Accordingly, the total resistance coefficient $C_{T}$ can be approximated by (33), with $R_{T}$ being the total resistance, $S$ the wetted surface, and the following coefficients: $C_{F}$ the frictional resistance, $C_{A}$ the incremental resistance, $C_{A A}$ air resistance, and $C_{R}$ the residual resistance. Applying the method from Kristensen et al. [21] the following estimations and calculations were made: (i) $S$ according to (34), where $\nabla$ denotes the displacement, (ii) $C_{F}$ by (35), where Re represents the Reynolds number and $v$ the kinematic viscosity, (iii) $C_{A}=0.0004$, given that the hull was not produced according to towing tank standards, and its surface roughness is more in line with larger vessels, (iv) $C_{A A}=0.00007$ given that the vessel has plenty of extensions on its deck, and finally (v) for the estimation of $C_{R}$, [70] provided diagrams based on the length-displacement ratio $M_{d}$, prismatic coefficient $C_{P}$ where $C_{M}$ denotes the midship coefficient, and the Froude number $F_{r}$-see (36) for their definitions. This diagram estimation, $C_{R_{d}}$, should be corrected for several factors, $\sum C_{R_{\text {corr }}}$ : the position of the centre of buoyancy, hull shape and form, bulbous bow shapes, and the $\frac{B}{T}$ ratio—see (37). Based on Appendix I from [21], $C_{R_{d}}=0.001$, and only a correction for $\frac{B}{T}$ was added by the following equation: $C_{R_{c o r r}, \frac{B}{T}}=0.16\left(\frac{B}{T}-2.5\right) \times 10^{-3}$.

$$
\begin{gathered}
C_{T}=C_{F}+C_{A}+C_{A A}+C_{R}=\frac{R_{T}}{0.5 \rho S u^{2}} \\
S=0.99\left(\frac{\nabla}{T}+1.9 L_{w l} T\right), \quad \nabla=\frac{M}{\rho}
\end{gathered}
$$




$$
\begin{gathered}
C_{F}=\frac{0.0075}{(\log (R e)-2)^{2}}, \quad R e=\frac{u L_{w l}}{v} \\
M_{d}=\frac{L_{w l}}{\nabla^{1 / 3}}, \quad C_{P}=\frac{C_{B}}{C_{M}}, \quad F_{r}=\frac{u}{\sqrt{g L_{w l}}} \\
C_{R}=C_{R_{d}}+\sum C_{R_{c o r r}}
\end{gathered}
$$

Table 4 lists the additional measured, calculated, or estimated parameters of the geometry of the Cogge. The measured draft of $0.22 \mathrm{~m}$ would equal a vessel weight of $623 \mathrm{~kg}$, and vice versa, the measured weight of $590 \mathrm{~kg}$ would generate a draft of $0.21 \mathrm{~m}$. The latter weight and draft have been used for the calculations in this study. The geometry of the Cogge, with a $C_{B}=0.95$, lies outside the boundaries of some parts of the empirical method of Kristensen et al. [21]. Consequently, it is not straightforward to read, or extrapolate, the right $C_{R_{d}}$ from the diagrams, or to apply all these suggested $C_{R}$ corrections; hence, only the $\frac{B}{T}$ correction was added. Considering these limitations, made assumptions, and the neglected additional corrections, the predicted damping forces by this empirical method can thus be reasonably assumed to serve as an underestimation of the actual damping forces. Calculating $R_{T}$ via (33) with $u=1.0 \frac{\mathrm{m}}{\mathrm{s}}, \rho=997 \frac{\mathrm{kg}}{\mathrm{m}^{3}}, v=1.15 \times 10^{-6} \frac{\mathrm{m}^{2}}{\mathrm{~s}}$ was used to define (38) which provides an under limit for the surge damping forces of the Cogge.

$$
D_{\operatorname{Emp}}(u)=11.82 u^{2}
$$

Table 4. Measured, calculated, or estimated parameters of the Cogge.

\begin{tabular}{lrrrrr}
\hline Parameter & Symbol & Measured & Calculated & Estimated & Units \\
\hline Draft & $\mathrm{T}$ & $0.22 \pm 0.02$ & 0.21 & - & {$[\mathrm{m}]$} \\
Length waterline & $L_{w l}$ & $4.75 \pm 0.02$ & - & - & {$[\mathrm{m}]$} \\
Mass & $\mathrm{m}$ & $590 \pm 10$ & 623 & - & {$[\mathrm{kg}]$} \\
Midship section coefficient & $C_{M}$ & - & - & $0.99[21]$ & {$[-]$} \\
Prismatic coefficient & $C_{P}$ & - & 0.96 & - & {$[-]$} \\
\hline
\end{tabular}

\subsubsection{Comparisons Based on Experimental Data of This Study}

In addition to the two external data comparisons of Section 3.4.1, this section briefly handles three approaches to compare the model structures in this data, based solely on the present experimental data. First, one can use both identification methods of Section 3.3 for the same model structures and see whether both methods independently achieve similar results, which might hint at a good underlying model structure. Second, one can compare the final cost of (24) between model structures for an identification method. Note that one should not compare these costs between identification methods, given their different cost function declarations. Third, for both identification methods, all the data have been split into a verification and a validation set. The former groups all the training missions, whereas the latter gathers the missions which were not used during the identification procedure. Table 5 lists these manually selected validation missions. These missions were chosen to be the missions with the highest initial conditions, i.e., vessel speeds. Accordingly, we aimed to offer a robust test set of data to investigate the model structures and identification methods of this study. This selection should also increase the performance of the FBM, which is more sensitive to these start conditions.

Table 5. Manually chosen validation missions.

\begin{tabular}{lcll}
\hline Surge & Sway & Yaw \\
\hline bow-ramp- $m_{2}$, & bow-step- $m_{1}$, & left-ramp- $m_{2}$, & cw-ramp- $m_{1}$, \\
stern-ramp- $m_{1}$, & stern-stairs- $m_{1}$, & left-stairs- $m_{2}$, & cw-step- $m_{2}$, \\
dual-ramp- $m_{2}$, & dual-stairs- $m_{2}$ & right-step- $m_{2}$ & ccw-stairs- $m_{1}$ \\
\hline
\end{tabular}




\section{Results}

Sections 4.1-4.3 respectively discuss the results for the surge, sway, and yaw motion models. Each section starts with plotting experimental data, continues with the identification and comparison of their model structures, and ends with a suggested model selection.

\subsection{Results of Surge Motion Models}

\subsubsection{Exemplary Data Surge Missions}

Figure 7 summarises the relevant measured data, which were processed according to Section 3.2.2, from the first-step mission with both thrusters on. Figure 7a reveals that the measured $n_{b}$ and $n_{s}$ overshot before they reached their requested values. This behaviour offers a richer data set, and can also be noted in the measured $\dot{u}$. Figure $7 \mathrm{~b}, \mathrm{c}$ confirms that the sway speeds remained small compared to the surge speeds, and that the heading did not change much during the mission.

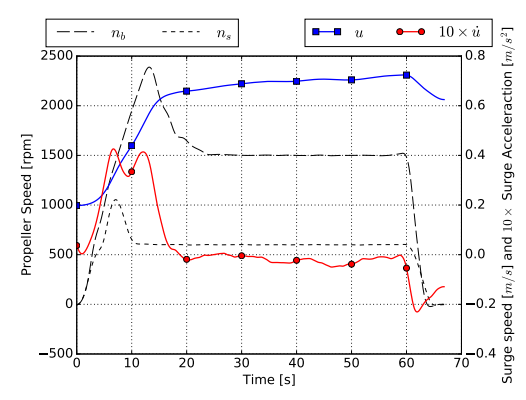

(a) Surge

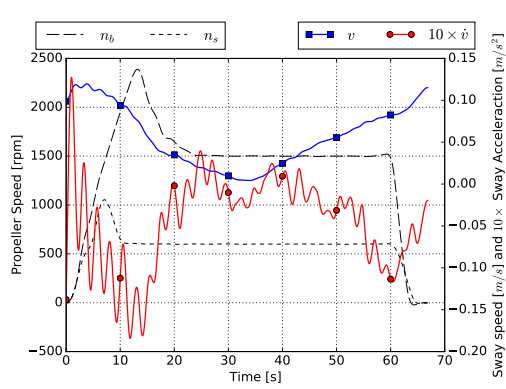

(b) Sway

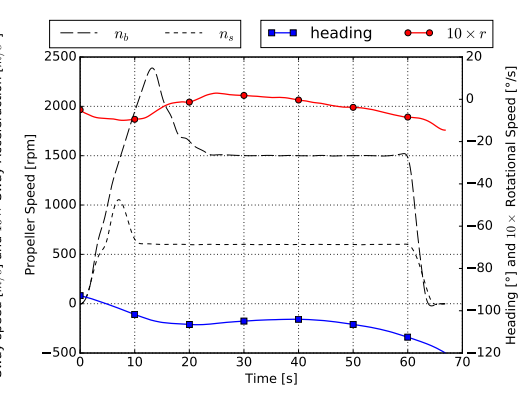

(c) Yaw

Figure 7. Data fetched during the first surge dual step mission: (a) surge speed and acceleration, (b) sway speed and acceleration, and (c) heading and yaw-rate.

\subsubsection{Surge Model Structures' Identification and Comparison}

The surge motion submode experiments enabled data to be recorded where the thrusters did not cooperate. These independent operation modes, in combination with the dual modes, might offer the most versatile data sets in this study. For this reason, all the surge models, $\mathcal{M}\left(\boldsymbol{\theta}_{u}^{x}\right)$, have been identified by both the FMB and DEM once using physical boundaries on the thruster parameters, and once with unbounded thruster coefficients, denoted by the superscripts ${ }^{B}$ and ${ }^{U}$ respectively. For both the bounded and unbounded training runs, the initial guesses $T_{n v_{0}}^{0, b}=T_{n n v_{0}}^{0, b}=T_{n v_{0}}^{0, s}=T_{n n v_{0}}^{0, s}=0$. The initial guesses for $T_{n n_{0}}^{0, b}=\frac{13.7}{(1500)^{2}} \frac{\mathrm{N}}{\mathrm{rpm}^{2}}$ and $T_{n n_{0}}^{0, \mathrm{~s}}=\frac{135}{(1545)^{2}} \frac{\mathrm{N}}{\mathrm{rpm}^{2}}$ were calculated based on the towing tank data by assuming a purely quadratic fit to the data points at the highest measured propeller speeds, see Figure A1. For the unbounded training, $T_{n v}^{0, b}, T_{n n v}^{0, b}, T_{n v}^{0, s}, T_{n n v}^{0, s} \in[0,-\infty]$, and $T_{n n}^{0, b}, T_{n n}^{0, s} \in[0,+\infty]$. For the bounded training $T_{n n}^{0, b} \in\left[0.85 \times T_{n n_{0}}^{0, b}, 1.15 \times T_{n n_{0}}^{0, b}\right]$, and $T_{n n}^{0, s} \in\left[0.25 \times T_{n n_{0}}^{0, s}, 1.10 \times T_{n n_{0}}^{0, s}\right]$. These boundaries aim to: (i) take into account the data uncertainty of both thrusters [22], (ii) allow some slack, given that higher rotational speeds were used compared to the towing tank data and the steering grid was now inside a hull, see Figure A1, and (iii) investigate the installed flow straightener, see [3], in the stern thruster which was not installed during the towing tank experiments. Finally, the $T_{n v}^{0, b}, T_{n n u}^{0, b}, T_{n v}^{0, s}, T_{n n u}^{0, s}$ had an upper limit of zero, and an lower limit which would induce a thrust deduction the size of the available thrust which was taken to be $85 \%$ of the initial guess. More precisely, these lower limits were calculated using the following data of two independent step missions: $n_{b}=$ $2700 \mathrm{rpm}, u=1.2 \frac{\mathrm{m}}{\mathrm{s}}$ for the bow, and $n_{s}=1400 \mathrm{rpm}, u=0.8 \frac{\mathrm{m}}{\mathrm{s}}$ for the stern.

The initial guesses and the boundaries for the added mass and damping models stayed the same throughout all model structures for both the bounded and unbounded methods. The initial guess of $X_{\dot{u}}=35.4 \frac{\mathrm{Ns}^{2}}{\mathrm{~m}}$ equalled the upper limit of (3). To potentially compensate for the weight measurement accuracy and the high $C_{B}$ of the Cogge, this added mass received the following training boundaries $X_{\dot{u}} \in[25,75]$. The present damping parameters received an initial value of seven and the training 
limits $X_{u u u}, X_{u u}, X_{u} \in[0,100]$. Evidently, if a damping or thruster parameter was not in $\theta_{u}^{x}$ it was not used during its model fit.

Table 6 summarises the residuals of the bounded and unbounded FBM and DEM cost functions for all the surge model structures, i.e., (25) and (29) for $x \in[a, \cdots, i]$. Table 7 zooms in on the $\mathrm{DEM}^{B}$ by listing its identified coefficients. The coefficients for $\mathrm{DEM}^{U}, \mathrm{FBM}^{B}$, and $\mathrm{FBM}^{U}$, can be respectively found in the Appendices Tables A1-A3. Based on these identified coefficients, Figure 8 plots the predicted speed profiles, simulated by the differential equations, for all the model structures with a quadratic damping model, i.e., $\mathcal{M}\left(\boldsymbol{\theta}_{u}^{b}\right), \mathcal{M}\left(\boldsymbol{\theta}_{u}^{e}\right)$, and $\mathcal{M}\left(\boldsymbol{\theta}_{u}^{h}\right)$, for three validation and three verification cases. The bias term for each mission was determined by retraining (25) or (29) for only one mission using the identified coefficients and only allowing $\beta_{u_{I}}$ to vary. For both the validation and the verification missions, the $\mathrm{FBM}^{B}$ seems to better capture the data compared to its unbounded alternative. For the bounded and unbounded DEM, there seems to be no significant difference, which can also be noted by the final costs of Table 6 . In general, if we judge the model structures on their ability to capture the shape of the data sets, the speed-independent thruster model, $\mathcal{M}\left(\boldsymbol{\theta}_{u}^{e}\right)$, looks to perform the worst, which Table 6 might be confirming, given that this model structure consistently has a higher final cost compared to $\mathcal{M}\left(\boldsymbol{\theta}_{\boldsymbol{u}}^{b}\right)$ and $\mathcal{M}\left(\boldsymbol{\theta}_{\boldsymbol{u}}^{h}\right)$ for all the listed identification approaches.

Table 6. Final costs for the surge identification methods.

\begin{tabular}{lrrrrrrrrr}
\hline & $\boldsymbol{\theta}_{u}^{a}$ & $\boldsymbol{\theta}_{u}^{\boldsymbol{b}}$ & $\boldsymbol{\theta}_{\boldsymbol{u}}^{\boldsymbol{c}}$ & $\boldsymbol{\theta}_{u}^{\boldsymbol{d}}$ & $\boldsymbol{\theta}_{u}^{\boldsymbol{e}}$ & $\boldsymbol{\theta}_{\boldsymbol{u}}^{f}$ & $\boldsymbol{\theta}_{u}^{g}$ & $\boldsymbol{\theta}_{u}^{\boldsymbol{h}}$ & $\boldsymbol{\theta}_{u}^{\boldsymbol{i}}$ \\
\hline $\mathrm{FBM}^{B}$ & $44,306.46$ & $44,460.31$ & $50,313.20$ & $79,170.60$ & $80,628.91$ & $121,466.91$ & $59,083.96$ & $59,675.91$ & $63,849.69$ \\
$\mathrm{FBM}^{U}$ & $43,361.09$ & $43,361.09$ & $44,946.84$ & $144,849.19$ & $85,623.04$ & $228,775.01$ & $59,083.96$ & $45,0793.14$ & $63,572.62$ \\
$\mathrm{DEM}^{B}$ & 13.69 & 14.36 & 18.25 & 35.64 & 45.28 & 92.83 & 19.13 & 20.77 & 23.67 \\
$\mathrm{DEM}^{U}$ & 13.69 & 14.24 & 15.31 & 36.19 & 45.33 & 92.83 & 19.14 & 20.79 & 23.69 \\
\hline
\end{tabular}

Table 7. Identified surge model coefficients for $\mathrm{DEM}^{B}$.

\begin{tabular}{|c|c|c|c|c|c|c|c|c|c|}
\hline & $\theta_{u}^{a}$ & $\theta_{u}^{b}$ & $\theta_{u}^{c}$ & $\theta_{u}^{d}$ & $\theta_{u}^{e}$ & $\theta_{u}^{f}$ & $\theta_{u}^{g}$ & $\theta_{u}^{h}$ & $\theta_{u}^{i}$ \\
\hline cost & 13.7 & 14.4 & 18.2 & 35.6 & 45.3 & 92.8 & 19.1 & 20.8 & 23.7 \\
\hline$X_{\dot{u}}$ & 25.7 & 25.0 & 25.0 & 75.0 & 75.0 & 58.6 & 30.5 & 39.8 & 27.7 \\
\hline$X_{\text {иии }}$ & 8.11 & 0.00 & 0.00 & 25.0 & 0.00 & 0.00 & 12.8 & 0.00 & 0.00 \\
\hline$X_{u и}$ & 0.00 & 11.0 & 0.00 & 0.00 & 36.3 & 0.00 & 0.00 & 13.6 & 0.00 \\
\hline$X_{u}$ & 14.7 & 10.8 & 19.0 & 15.9 & 3.77 & 39.3 & 17.6 & 14.0 & 22.9 \\
\hline$T_{n n}^{0, b}$ & $6.92 \times 10^{-6}$ & $7.00 \times 10^{-6}$ & $7.00 \times 10^{-6}$ & $6.03 \times 10^{-6}$ & $6.02 \times 10^{-6}$ & $5.42 \times 10^{-6}$ & $5.50 \times 10^{-6}$ & $6.21 \times 10^{-6}$ & $6.99 \times 10^{-6}$ \\
\hline$T_{n n}^{0, s}$ & $2.62 \times 10^{-5}$ & $2.66 \times 10^{-5}$ & $2.75 \times 10^{-5}$ & $1.69 \times 10^{-5}$ & $1.62 \times 10^{-5}$ & $1.68 \times 10^{-5}$ & $2.58 \times 10^{-5}$ & $2.71 \times 10^{-5}$ & $2.81 \times 10^{-5}$ \\
\hline$T_{n v}^{0, b}$ & $-7.02 \times 10^{-3}$ & $-7.54 \times 10^{-3}$ & $-8.79 \times 10^{-3}$ & 0.00 & 0.00 & 0.00 & 0.00 & 0.00 & 0.00 \\
\hline$T_{n v}^{0, s}$ & $-2.67 \times 10^{-2}$ & $-2.78 \times 10^{-2}$ & $-3.00 \times 10^{-2}$ & 0.00 & 0.00 & 0.00 & 0.00 & 0.00 & 0.00 \\
\hline$T_{n n v}^{0, b}$ & 0.00 & 0.00 & 0.00 & 0.00 & 0.00 & 0.00 & $-3.55 \times 10^{-7}$ & $-1.42 \times 10^{-6}$ & $-2.98 \times 10^{-6}$ \\
\hline$T_{n n v}^{0, s}$ & 0.00 & 0.00 & 0.00 & 0.00 & 0.00 & 0.00 & $-1.52 \times 10^{-5}$ & $-1.70 \times 10^{-5}$ & $-1.89 \times 10^{-5}$ \\
\hline
\end{tabular}




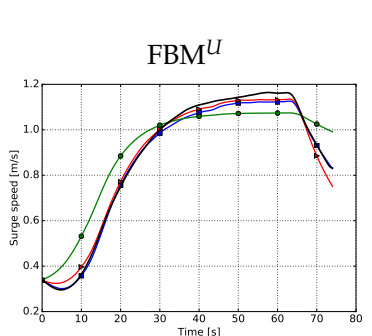

(a)

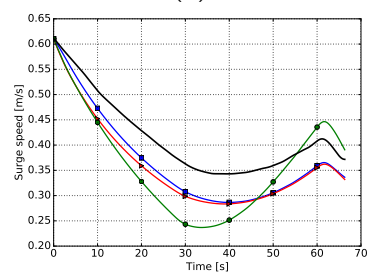

(e)

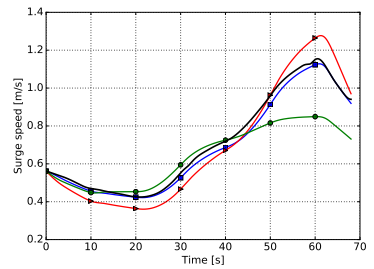

(i)

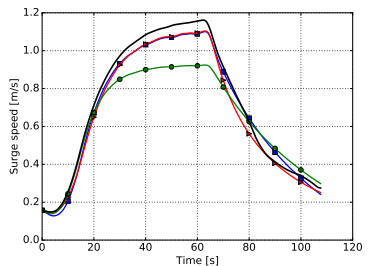

(m)

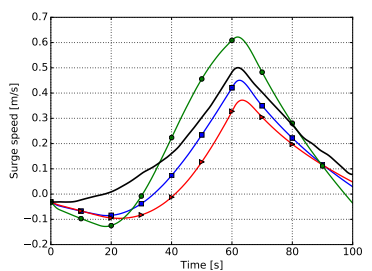

(q)

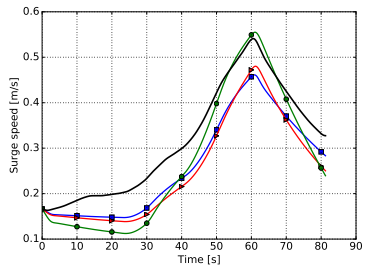

(u)

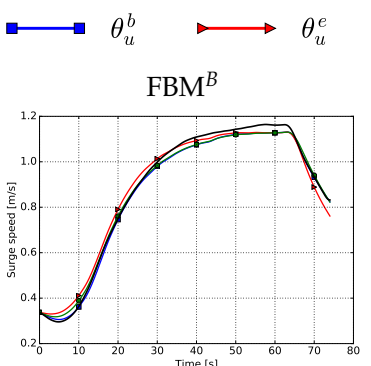

(b)

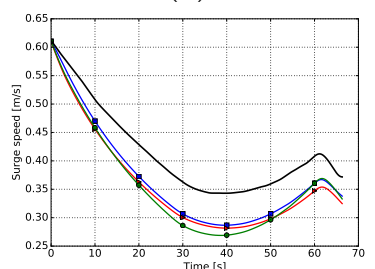

(f)

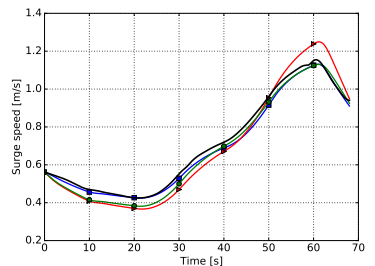

(j)

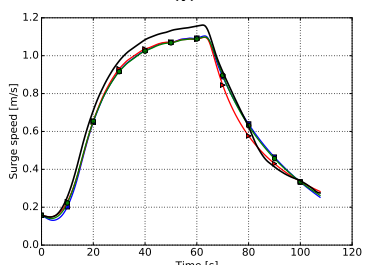

(n)

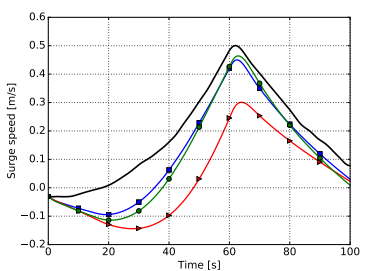

(r)

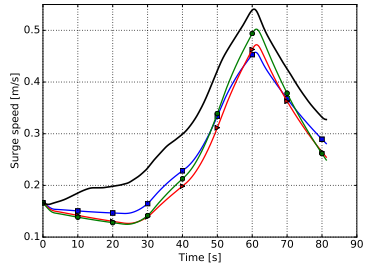

(v)

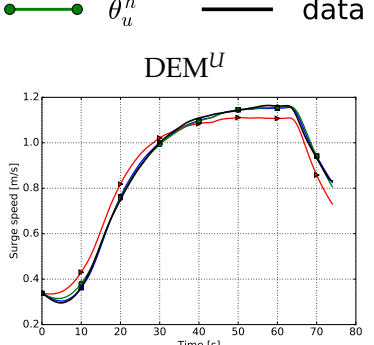

(c)

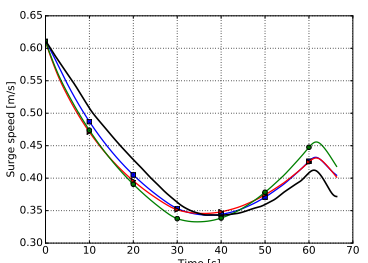

(g)

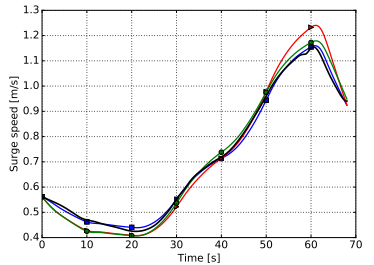

(k)

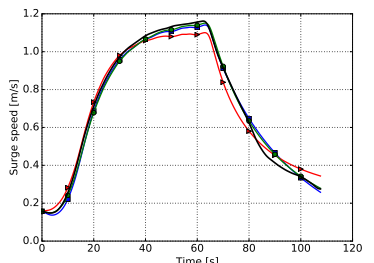

(o)

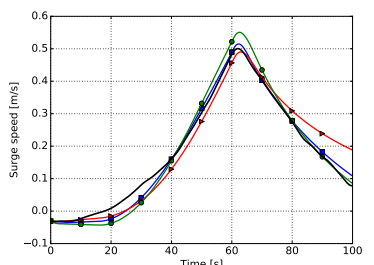

(s)

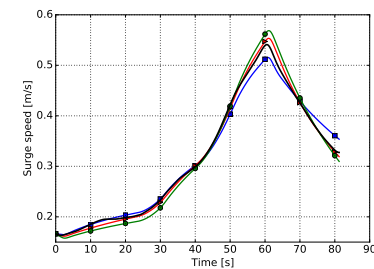

(w)

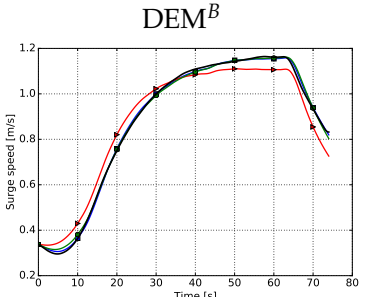

(d)

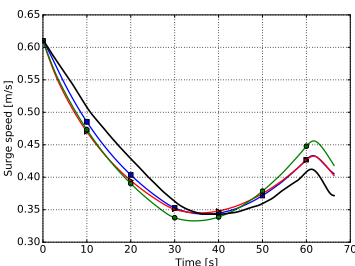

(h)

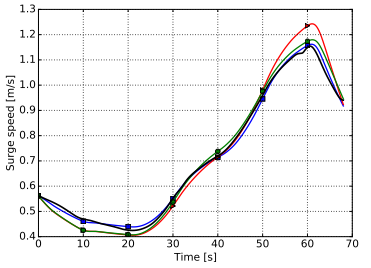

(1)

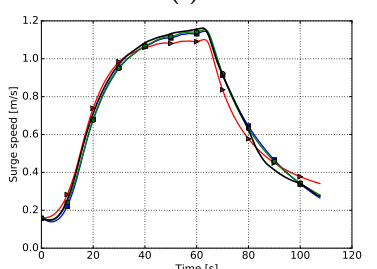

(p)

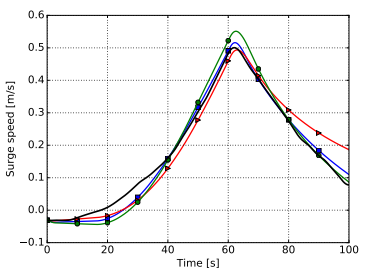

(t)

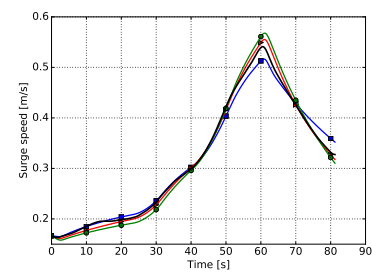

$(\mathbf{x})$

Figure 8. Comparison of bounded and unbounded FBM and DEM identification methods for the different thruster models with quadratic damping models: (a-d) validation mission: bow-step- $m_{1}$; $(\mathbf{e}-\mathbf{h})$ validation mission: stern-ramp- $m_{1} ;(\mathbf{i}-\mathbf{l})$ validation mission: dual-stairs $-m_{2} ;(\mathbf{m}-\mathbf{p})$ verification mission: bow-step- $m_{2} ;(\mathbf{q}-\mathbf{t})$ verification mission: stern-ramp- $m_{2} ;$ and $(\mathbf{u}-\mathbf{x})$ verification mission: dual-stairs $-m_{1}$. 


\subsubsection{Surge Model Selection}

The identification results of Section 4.1.2 can also be compared with the external data from Section 3.4.1. Accordingly, Figure 9a-d compares the identified cubic damping models for the three different thruster models, i.e., $\mathcal{M}\left(\boldsymbol{\theta}_{u}^{\boldsymbol{a}}\right), \mathcal{M}\left(\boldsymbol{\theta}_{\boldsymbol{u}}^{\boldsymbol{d}}\right)$, and $\mathcal{M}\left(\boldsymbol{\theta}_{\boldsymbol{u}}^{\boldsymbol{g}}\right)$, with the curves defined by (32) and (38). The damping models for the speed-independent thruster model seem to be consistently too large for both the bounded and unbounded FBMs and DEMs. Figure 9e-h similarly contrasts the identified bollard thrust forces of the bow thruster together with its experimental data. Note that this comparison was not made for the stern thruster due to its installed flow straightener which would make it an unfair benchmark. Evidently, the bollard thrust forces for the bounded FBM and DEM stay close to the experimental data, however, both unbounded methods do stay within a close range too, which might hint at a good model structure and/or identification methodology. Finally, given that the conventional thruster model seems to perform well according to Table 6 and Figures 8 and 9 , in both DEM ${ }^{B}$ and $\mathrm{DEM}^{U}$, Figure 10 illustrates the three different damping models for this thruster model. In particular, Figure 10a-c shows the simulated the model responses for the three validation cases, Figure 10d compares the damping forces with the curves from (32) and (38), and Figure 10e,f predicts the bollard thruster forces.

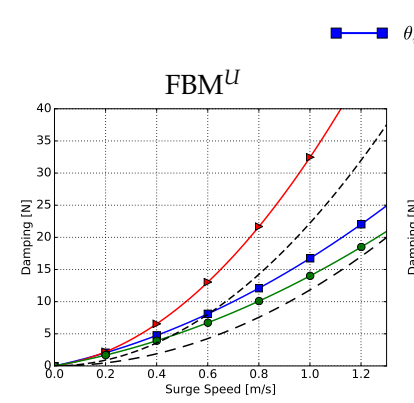

(a)

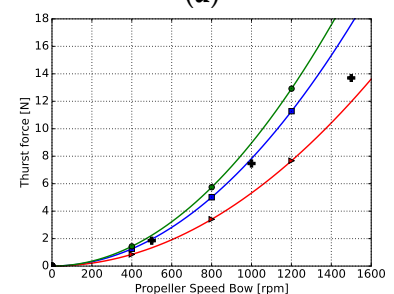

(e)

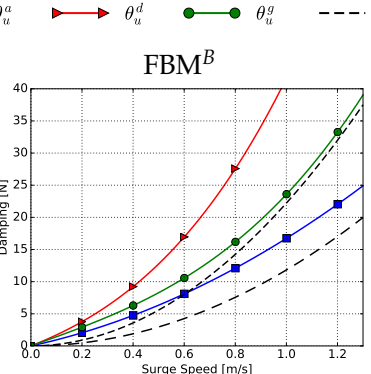

(b)

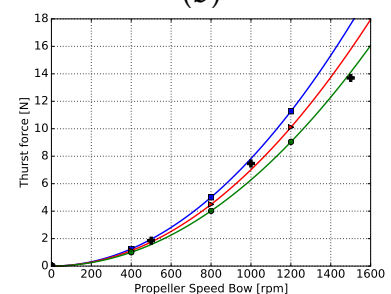

(f)

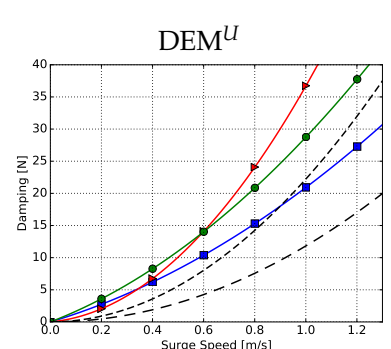

(c)

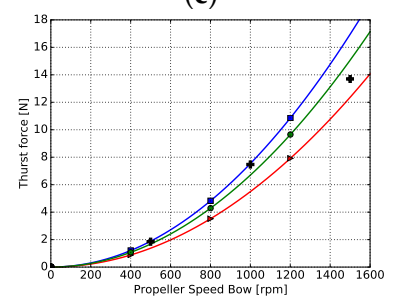

(g)

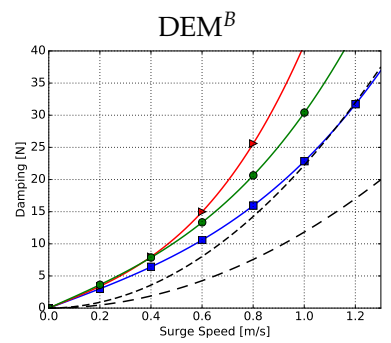

(d)

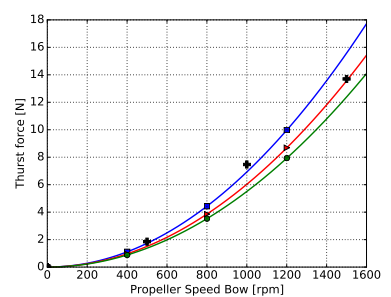

(h)

Figure 9. Comparison of bounded and unbounded FBM and DEM surge identification methods for the cubic damping models: (a-d) predicted damping models in comparison with Peeters et al. [20], i.e., (32) and Kristensen et al. [21]—(38); and (e-h) predicted bollard pull forces of the bow thruster in comparison with the towing tank data from Peeters et al. [22]. 


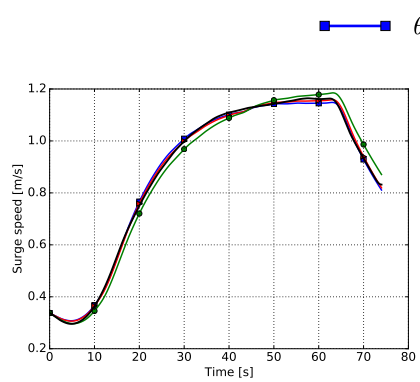

(a) bow-step- $m_{1}$

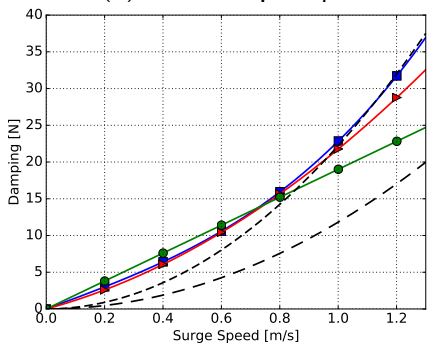

(d) Damping force

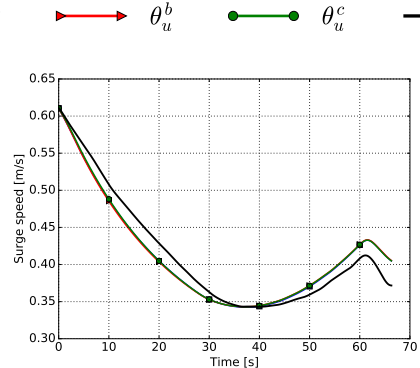

(b) stern-ramp- $m_{1}$

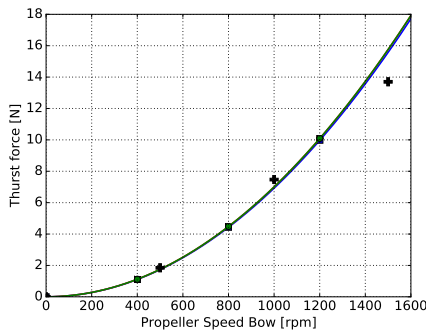

(e) Thrust Force Bow data

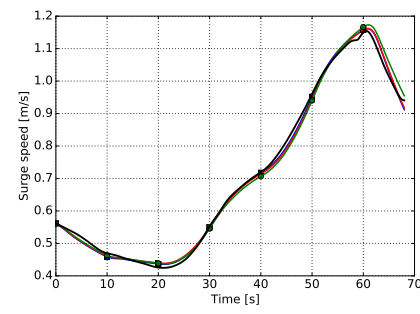

(c) dual-stairs- $m_{2}$

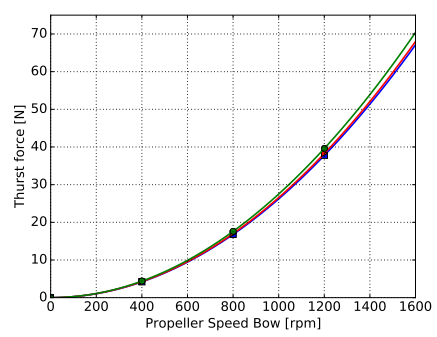

(f) Thrust Force Stern $\longrightarrow \begin{array}{lll}\longrightarrow \theta_{u}^{a} & -- \text { CFD Peeters et al. } \\ \theta_{u}^{b} & -- & \text { Kristensen et al. }\end{array}$

Figure 10. Comparisons of different damping models for the conventional thruster model, identified with the $\mathrm{DEM}^{B}:(\mathbf{a}-\mathbf{c})$ the three longitudinal validation missions, (d) the predicted damping forces compared with Peeters et al. [20], i.e., (32), and Kristensen et al. [21]-(38); (e) predicted bollard thrust bow compared with towing tank data from Peeters et al. [22], and (f) predicted bollard stern thrust with installed flow straightener; hence the towing tank data was not plotted.

\subsection{Results of Sway Motion Models}

\subsubsection{Exemplary Data Sway Missions}

Figure 11 outlines the relevant captured data, which were processed according to Section 3.2.2, from the first ramp mission where the vessel swayed to the right. This figure displays that both propellers have a lower limit on their rotational speed, hence the straight parts in the ramp profiles of the propeller speeds. Figure 11c reveals that although the heading oscillated a bit during the mission the vessel stayed rather straight. Finally, Figure 11a shows a rather small surge speed compared to the sway speed of Figure 11b.

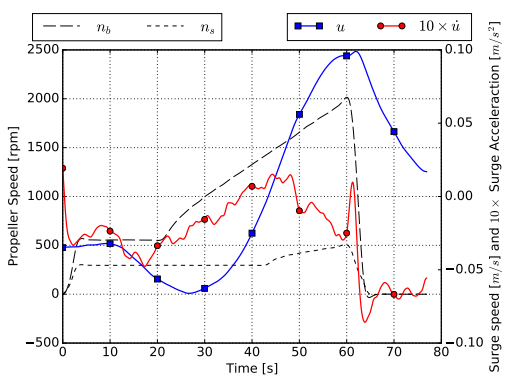

(a) Surge

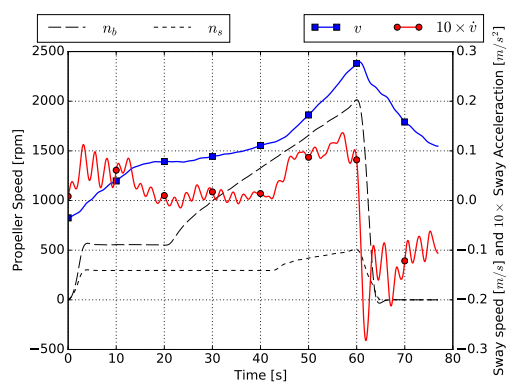

(b) Sway

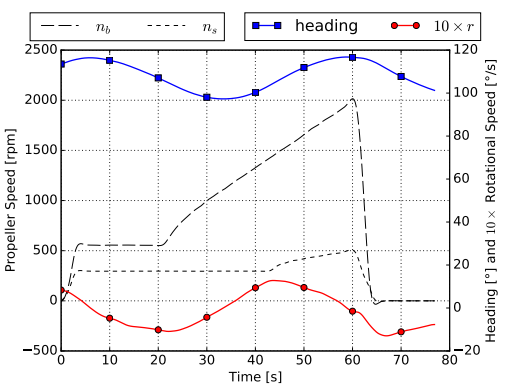

(c) Yaw

Figure 11. Data fetched during the first transversal right ramp mission: (a) surge speed and acceleration, (b) sway speed and acceleration, and (c) heading and yaw-rate. 


\subsubsection{Sway Model Structures' Identification and Comparison}

Considering the good $\mathrm{DEM}^{B}$ performance in Section 4.1, these results for the sway model identifications will be exclusively discussed here. The determination of the initial parameter estimations and their training boundaries happened similarly to the surge model approach. Thus, according to (4), the added mass $Y_{\dot{v}}=383 \frac{\mathrm{Ns}^{2}}{\mathrm{~m}}$, with the limits $Y_{\dot{v}} \in[373,583]$. The damping terms started at 500 and had the boundaries of $Y_{v v v}, Y_{v v}, Y_{v} \in\left[0,1.0 \times 10^{4}\right]$. Taking into consideration that (i) the surge experiments had the most versatile data sets, (ii) Section 3.1.4 assumed the surge or sway wake effect differences to be negligible, and (iii) both the bounded and unbounded FBMs and DEMs identified speed-dependent thruster coefficients in the same order of magnitude, the start estimations for the coefficients, $T_{n v_{0}}^{90, b}, T_{n n v_{0}}^{90, b}$, and $T_{n v_{0}}^{90, s}, T_{n n v_{0}}^{90, s}$, were selected to equal the values of their $\mathrm{DEM}^{b}$ identified $T\left(0^{\circ}\right)$ equivalents for surge; see Table 7 . In order to consider the angle dependency of the thrust deduction (see Figure A1), $t$, lower and upper limits of $90 \%$ and $110 \%$ of their initial value were given to these parameters. The initial guesses for $T_{n n_{0}}^{90, b}=\frac{14.52}{(1500)^{2}} \frac{\mathrm{N}}{r p m^{2}}$ and $T_{n n_{0}}^{90, s}=\frac{26.15}{(617)^{2}} \frac{\mathrm{N}}{r p m^{2}}$ were calculated based on the towing tank data by assuming a purely quadratic fit to the highest data points closest to the highest propeller speed of the experiments; see Figure A1. Here too, upper and lower limits of $90 \%$ and $110 \%$ were implemented for both thrusters. These boundaries aimed to: (i) take into account the data uncertainty of both thrusters [22], and (ii) allow some slack, given that higher rotational speeds were used compared to the towing tank data and the steering grid was now inside a hull, see Figure A1.

Table 8 states the $\mathrm{DEM}^{B}$ identified coefficients for $\mathcal{M}\left(\boldsymbol{\theta}_{v}^{\boldsymbol{x}}\right)$. Thereupon, Figure 12 predicts the sway speed profiles for three validation and three verification missions for the quadratic damping models, i.e., $\mathcal{M}\left(\boldsymbol{\theta}_{v}^{b}\right), \mathcal{M}\left(\boldsymbol{\theta}_{v}^{e}\right)$, and $\mathcal{M}\left(\boldsymbol{\theta}_{v}^{h}\right)$. The bias terms for each missions were determined similar to the surge speed predictions of Section 4.1.2, and the negative initial sway speeds of two of the validation missions were taken into account in their differential equations. Both Table 8 and Figure 12 indicate minor differences between the different model structures.

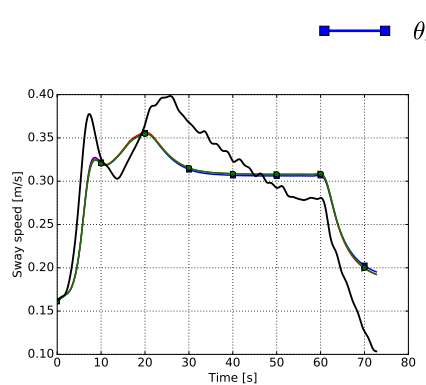

(a) right-step- $m_{2}$

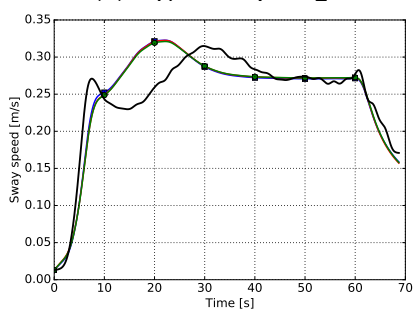

(d) right-step- $m_{1}$

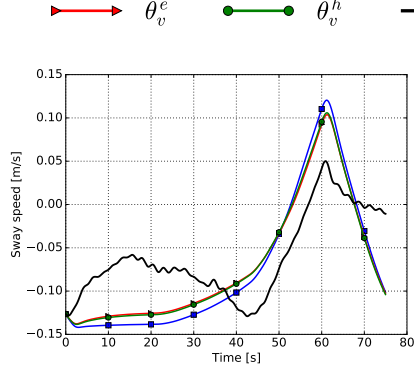

(b) left-ramp- $m_{2}$

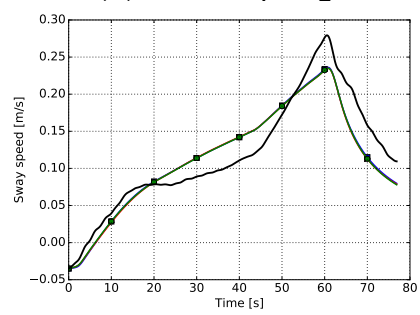

(e) right-ramp- $m_{1}$

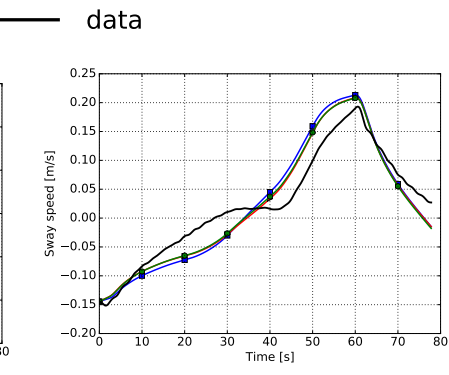

(c) left-stairs- $m_{2}$

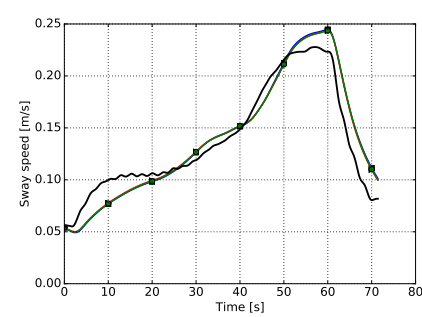

(f) left-stairs- $m_{1}$

Figure 12. Comparison of different thruster models using the quadratic damping model: $(\mathbf{a}-\mathbf{c})$ the validation missions and $(\mathbf{d}-\mathbf{f})$ the verification missions. 
Table 8. Identified sway model coefficients for the bounded DEM.

\begin{tabular}{|c|c|c|c|c|c|c|c|c|c|}
\hline & $\theta_{v}^{a}$ & $\theta_{v}^{b}$ & $\theta_{v}^{c}$ & $\theta_{v}^{d}$ & $\theta_{v}^{e}$ & $\theta_{v}^{f}$ & $\theta_{v}^{g}$ & $\theta_{v}^{h}$ & $\theta_{v}^{i}$ \\
\hline cost & 10.1 & 10.6 & 12.8 & 10.4 & 11.0 & 14.1 & 10.4 & 10.8 & 12.6 \\
\hline$Y_{\dot{v}}$ & 430.26 & 440.51 & 583.00 & 569.82 & 583.00 & 583.00 & 547.97 & 545.61 & 583.00 \\
\hline$Y_{v v v}$ & 1424.59 & 0.00 & 0.00 & 1732.56 & 0.00 & 0.00 & 1663.00 & 0.00 & 0.00 \\
\hline$Y_{v v}$ & 0.00 & 557.57 & 0.00 & 0.00 & 682.70 & 0.00 & 0.00 & 616.06 & 0.00 \\
\hline$Y_{v}$ & 56.43 .7 & 1.70 & 173.44 & 68.62 & 3.26 & 226.31 & 67.87 & 9.1 & 195.21 \\
\hline$T_{n n}^{90, b}$ & $5.81 \times 10^{-6}$ & $5.81 \times 10^{-6}$ & $5.81 \times 10^{-6}$ & $5.81 \times 10^{-6}$ & $5.81 \times 10^{-6}$ & $5.81 \times 10^{-6}$ & $5.81 \times 10^{-6}$ & $5.81 \times 10^{-6}$ & $5.81 \times 10^{-6}$ \\
\hline$T_{n n}^{90, s}$ & $7.56 \times 10^{-5}$ & $7.56 \times 10^{-5}$ & $7.56 \times 10^{-5}$ & $7.56 \times 10^{-5}$ & $7.56 \times 10^{-5}$ & $7.56 \times 10^{-5}$ & $7.56 \times 10^{-5}$ & $7.56 \times 10^{-5}$ & $7.56 \times 10^{-5}$ \\
\hline$T_{n v}^{90, b}$ & $-7.72 \times 10^{-3}$ & $-8.29 \times 10^{-3}$ & $-9.67 \times 10^{-3}$ & 0.00 & 0.00 & 0.00 & 0.00 & 0.00 & 0.00 \\
\hline$T_{n v}^{90, s}$ & $-2.40 \times 10^{-2}$ & $-2.50 \times 10^{-2}$ & $-3.30 \times 10^{-2}$ & 0.00 & 0.00 & 0.00 & 0.00 & 0.00 & 0.00 \\
\hline$T_{n n v}^{90, b}$ & 0.00 & 0.00 & 0.00 & 0.00 & 0.00 & 0.00 & $-3.91 \times 10^{-07}$ & $-1.56 \times 10^{-6}$ & $-3.28 \times 10^{-6}$ \\
\hline$T_{n n v}^{90, s}$ & 0.00 & 0.00 & 0.00 & 0.00 & 0.00 & 0.00 & $-1.37 \times 10^{-5}$ & $-1.53 \times 10^{-5}$ & $-1.70 \times 10^{-5}$ \\
\hline
\end{tabular}

\subsubsection{Sway Model Selection}

With the currently available training data, no model structure seems to outperform its competitors. Figure 13a-c depicts the sway speed predictions for three verification missions for the different damping models using the conventional thruster model. These figures indicate little differences between the linear, quadratic, or cubic damping models. Figure 13d predicts these damping forces, and Figure 13e,f evaluates the bollard pull forces for the bow and stern thruster in comparison with their towing tank data.

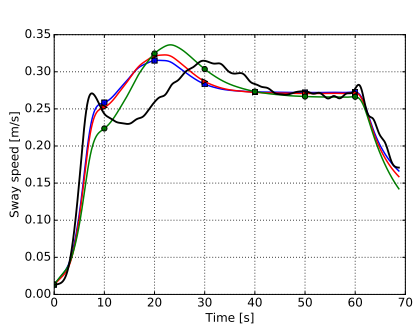

(a) right-step- $m_{1}$

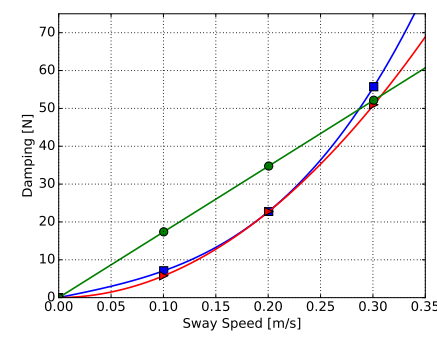

(d) Damping force

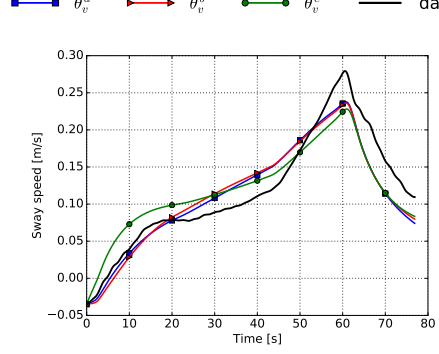

(b) right-ramp- $m_{1}$

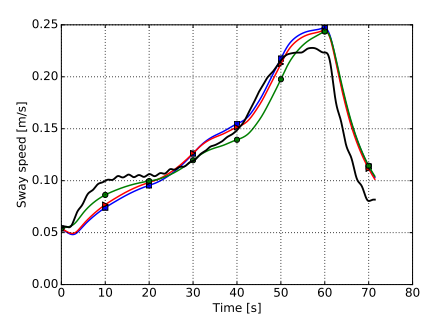

(c) left-stairs- $m_{1}$

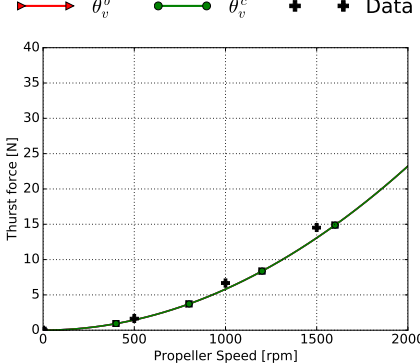

(e) Thrust force bow

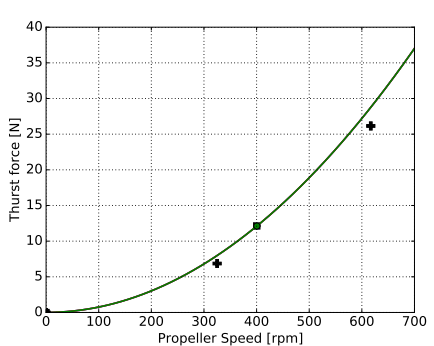

(f) Thrust force stern.

Figure 13. Comparisons of different damping models for the conventional thruster model, identified with the bounded DEM: $(\mathbf{a}-\mathbf{c})$ the three verification missions, (d) the predicted damping forces, and $(\mathbf{e}, \mathbf{f})$ respectively show the identified bollard thrusts of the bow and stern thrusters compared with their towing tank data from Peeters et al. [22].

\subsection{Results of Yaw Motion Models}

\subsubsection{Exemplary Data Yaw Missions}

Figure 14 gives an overview of the relevant captured data, which were processed according to Section 3.2.2, from both stairs missions where the Cogge rotated counter clockwise. The trajectory plots of Figure 14b,d demonstrate that there was less wind during the first run of the experiments compared with the second run, given that for the same inputs, the trajectory of the Cogge during $m_{2}$ was more spread out in the diagonal XY-direction. Nevertheless, Figure 14a,c illustrates that similar yaw-rates were achieved during both missions. 


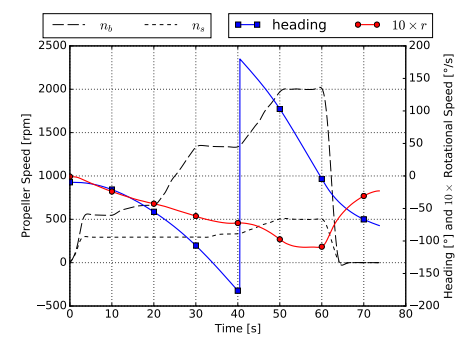

(a) Yaw- $m_{1}$

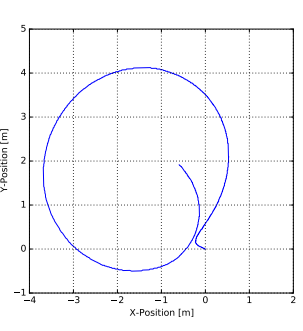

(b) Trajectory $-m_{1}$

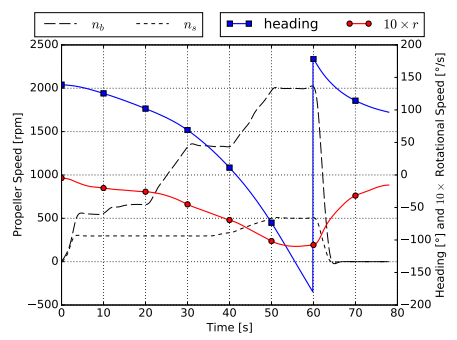

(c) Yaw $-m_{2}$

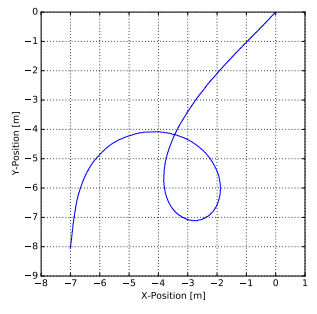

(d) Trajectory $-m_{2}$

Figure 14. Data fetched during the first and second rotation counter-clockwise stairs missions: $(\mathbf{a}, \mathbf{c})$ heading and yaw rate, and $(\mathbf{b}, \mathbf{d})$ position of the stern GNSS antenna.

\subsubsection{Yaw Model Structures' Identification and Comparison}

Since the yaw experiments used the same thruster angles and propeller speed configurations as the sway experiments, the exact same initial conditions and parameter boundaries were used for these thruster coefficients. The yaw moment of inertia estimation, $I_{z}=m(0.2536 \mathrm{~L})^{2}=878 \mathrm{kgm}^{2}$, followed the approach documented in [18,71]. The initial guess $N_{\dot{r}}=845 \frac{\mathrm{Nms}^{2}}{\mathrm{rad}}$, based on (5), received the boundaries $N_{\dot{r}} \in[845,1245]$. In addition, all the damping parameters started at 500 and were bounded to $N_{r r r}, N_{r r}, N_{r} \in\left[0,1.0 \times 10^{04}\right]$. Table 9 lists the coefficients for $\mathcal{M}\left(\boldsymbol{\theta}_{r}^{x}\right)$, identified by the $\mathrm{DEM}^{B}$. Subsequently, Figure 15 shows simulations of the yaw rates for three validation and three verification missions for the quadratic damping models, i.e., $\mathcal{M}\left(\boldsymbol{\theta}_{r}^{b}\right), \mathcal{M}\left(\boldsymbol{\theta}_{r}^{e}\right)$, and $\mathcal{M}\left(\boldsymbol{\theta}_{r}^{h}\right)$. Note that the yaw missions have no bias terms. In the same vein as the sway results of Section 4.2.2, both Table 9 and Figure 15 suggest minor differences between the different yaw model structures.

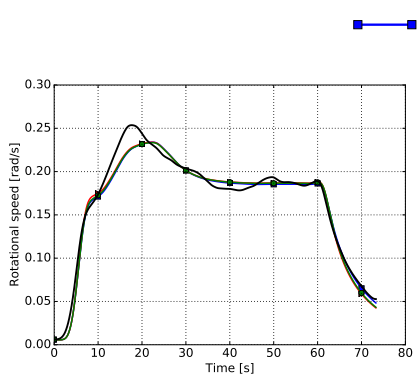

(a) cw-step- $m_{2}$

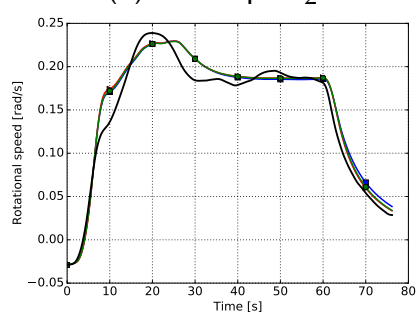

(d) ccw-step- $m_{2}$

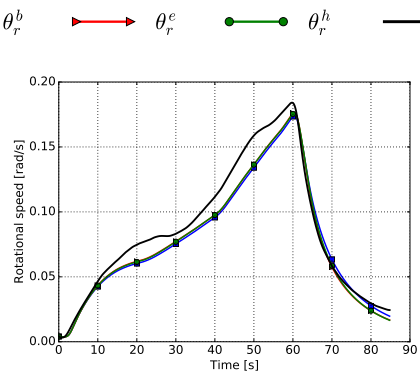

(b) cw-ramp- $m_{1}$

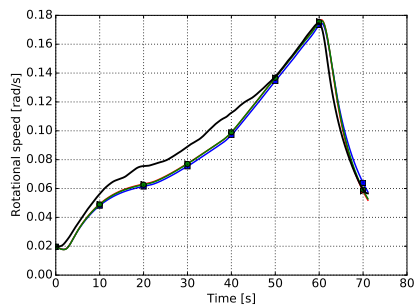

(e) ccw-ramp- $m_{1}$

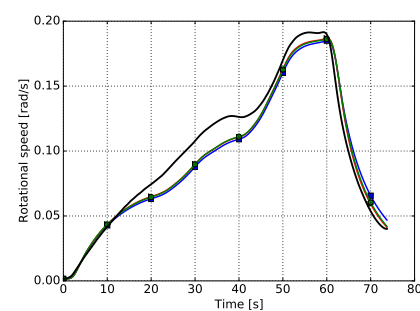

(c) ccw-stairs- $m_{1}$

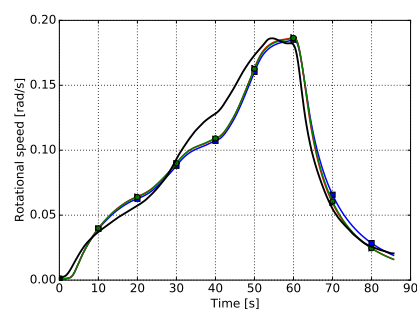

(f) cw-stairs- $m_{2}$

Figure 15. Comparison of different thruster models for the quadratic damping model: $(\mathbf{a}-\mathbf{c})$ the validation missions and $(\mathbf{d}-\mathbf{f})$ the verification missions. 
Table 9. Identified yaw model coefficients for the bounded DEM.

\begin{tabular}{|c|c|c|c|c|c|c|c|c|c|}
\hline & $\theta_{r}^{a}$ & $\theta_{r}^{b}$ & $\theta_{r}^{c}$ & $\theta_{r}^{d}$ & $\theta_{r}^{e}$ & $\theta_{r}^{f}$ & $\theta_{r}^{g}$ & $\theta_{r}^{h}$ & $\theta_{r}^{i}$ \\
\hline cost & 2.64 & 2.90 & 7.41 & 2.4 & 2.67 & 10.72 & 2.39 & 2.68 & 8.14 \\
\hline$N_{\dot{r}}$ & 845.00 & 845.00 & 845.00 & 887.07 & 877.95 & 969.59 & 845.00 & 845.00 & 845.00 \\
\hline$N_{r r r}$ & 4158.99 & 0.00 & 0.00 & 5278.05 & 0.00 & 0.00 & 4873.16 & 0.00 & 0.00 \\
\hline$N_{r r}$ & 0.00 & 1067.49 & 0.00 & 0.00 & 1282.86 & 0.00 & 0.00 & 1218.23 & 0.00 \\
\hline$N_{r}$ & 153.98 & 97.57 & 252.29 & 175.66 & 102.54 & 342.74 & 173.49 & 108.28 & 292.55 \\
\hline$T_{n n}^{90, b}$ & $7.10 \times 10^{-6}$ & $7.10 \times 10^{-6}$ & $5.81 \times 10^{-6}$ & $7.10 \times 10^{-6}$ & $7.10 \times 10^{-6}$ & $5.81 \times 10^{-6}$ & $7.10 \times 10^{-6}$ & $7.10 \times 10^{-6}$ & $5.81 \times 10^{-6}$ \\
\hline$T_{n n}^{90, s}$ & $6.18 \times 10^{-5}$ & $6.22 \times 10^{-5}$ & $7.56 \times 10^{-5}$ & $6.18 \times 10^{-5}$ & $6.18 \times 10^{-5}$ & $7.56 \times 10^{-5}$ & $6.18 \times 10^{-5}$ & $6.18 \times 10^{-5}$ & $7.27 \times 10^{-5}$ \\
\hline$T_{n v}^{90, b}$ & $-6.32 \times 10^{-3}$ & $-6.79 \times 10^{-3}$ & $-9.67 \times 10^{-3}$ & 0.00 & 0.00 & 0.00 & 0.00 & 0.00 & 0.00 \\
\hline$T_{n v}^{90, s}$ & $-2.40 \times 10^{-2}$ & $-2.50 \times 10^{-2}$ & $-3.30 \times 10^{-2}$ & 0.00 & 0.00 & 0.00 & 0.00 & 0.00 & 0.00 \\
\hline$T_{n n v}^{90, b}$ & 0.00 & 0.00 & 0.00 & 0.00 & 0.00 & 0.00 & $-3.20 \times 10^{-7}$ & $-1.28 \times 10^{-6}$ & $-3.28 \times 10^{-6}$ \\
\hline$T_{n n v}^{90, s}$ & 0.00 & 0.00 & 0.00 & 0.00 & 0.00 & 0.00 & $-1.67 \times 10^{-5}$ & $-1.87 \times 10^{-5}$ & $-2.08 \times 10^{-5}$ \\
\hline
\end{tabular}

\subsubsection{Yaw Model Selection}

Comparable with Section 4.2.3, no $\mathcal{M}\left(\boldsymbol{\theta}_{r}^{x}\right)$ seems to capture the yaw-rate data better than its alternatives. Figure 16a-c compares the conventional thruster model for the three damping models on the three validation missions. These comparisons indicate a better curve fitting for the non-linear damping models. Finally, Figure 16d illustrates the different predicted damping moments, and Figure 16e,f plots the computed bollard thrust forces for the bow and stern thruster.

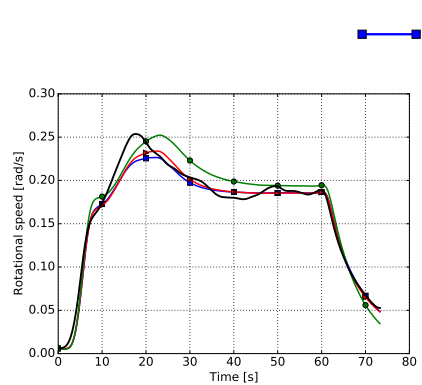

(a) cw-step- $m_{2}$

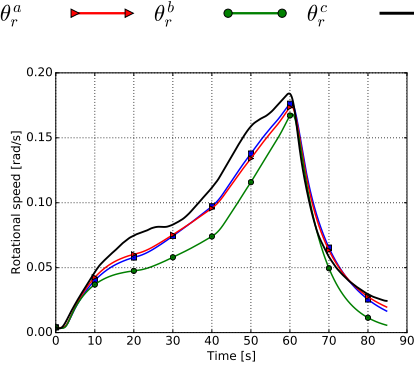

(b) cw-ramp- $m_{1}$

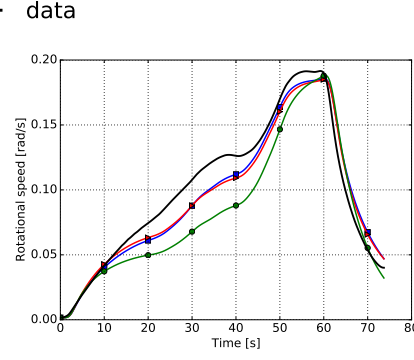

(c) ccw-stairs $-m_{1}$

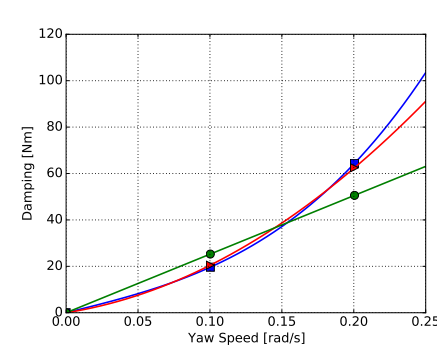

(d) Damping moment

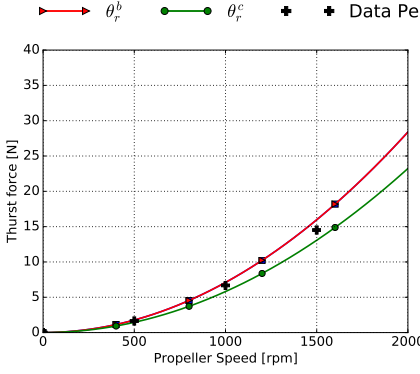

(e) Thrust force bow

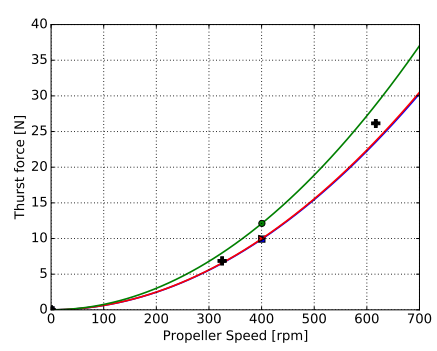

(f) Thrust force stern

Figure 16. Comparisons of different damping models for the conventional thruster model, identified with the bounded DEM: $(\mathbf{a}-\mathbf{c})$ the three validation missions, $(\mathbf{d})$ the predicted damping moments, and $(\mathbf{e}, \mathbf{f})$ respectively show the identified bollard thrusts of the bow and stern thruster compared with their towing tank data from Peeters et al. [22].

\section{Discussion}

When investigating the results of Section 4, the modelling assumptions and limitations of Section 3.1.1 need to be kept in mind. The provided exemplary mission data sets-see Sections 4.1.1, 4.2.1, and 4.3.1-already aided to judge the adequacy of these assumptions. In addition, Figure 17 sheds more light on the neglected degrees of freedom, i.e., roll, pitch, and heave, for three-step missions of the studied motions of surge, sway, and yaw. This figure reveals that the pitch angle did not vary much for these missions. The roll angle varied more compared to this pitch angle, and even changed almost $3^{\circ}$ during the rotational manoeuvre. The plotted heave represents the motions of 
the IMU which was positioned approximately $1.3 \mathrm{~m}$ behind the middle of the vessel [27]. Evidently, these neglected degrees of freedom do impact the hydrodynamics of the vessel.

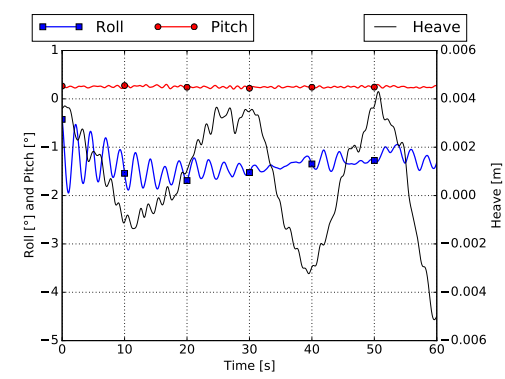

(a) dual-step- $m_{1}$

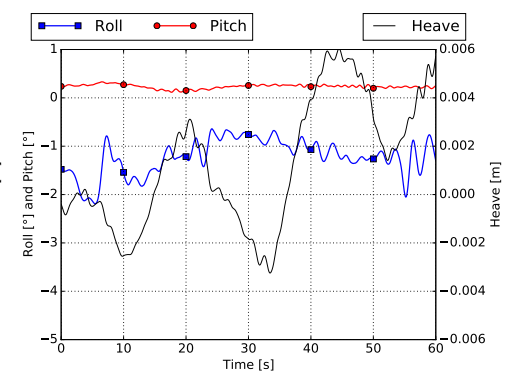

(b) right-step- $m_{1}$

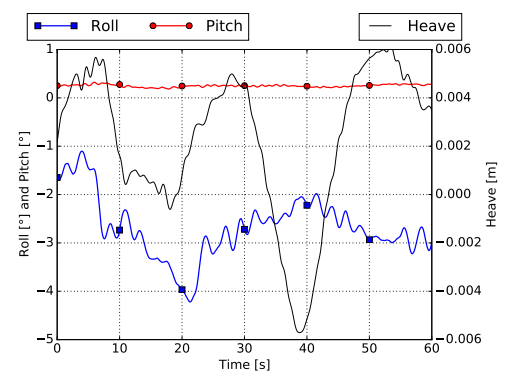

(c) ccw-stairs- $m_{1}$

Figure 17. Roll, pitch, and heave for a three-step mission in the three different motion modes: (a) dual-step- $m_{1}$, (b) right-step- $m_{1}$, and (c) ccw-stairs- $m_{1}$.

Bearing the just-mentioned assumptions and limitations in mind, the conventional thruster models, i.e., $\mathcal{M}\left(\boldsymbol{\theta}_{u}^{a}\right), \mathcal{M}\left(\boldsymbol{\theta}_{u}^{b}\right)$, and $\mathcal{M}\left(\boldsymbol{\theta}_{u}^{c}\right)$, seem to outperform their alternative thruster models for the surge motion according to: (i) their cost function results for both the FBM and the DEM-see Table 6; (ii) their predictions of both validation and verification missions-see Figure 8; and (iii) their comparison with the external CFD and empirical methods-see Figures 9 and 10. This combination of evidence might hint that these model structures best capture the occurring hydrodynamics. However, for the yaw and sway motion, there seems to be no real significant performance difference between the different thruster models. Accordingly, Figure 18 attempts to provide more insights into why this might be the case, by plotting the longitudinal or transversal pseudo-advanced speed ratios for surge, sway, and yaw step missions. The vessel speed was taken as advance speed to calculate these ratios; however, the propeller itself does not move in this speed direction, hence the name pseudo-advanced speed ratio. It can be seen that both thrusters achieved higher pseudo-advanced speed ratios in surge than in sway or yaw motion.

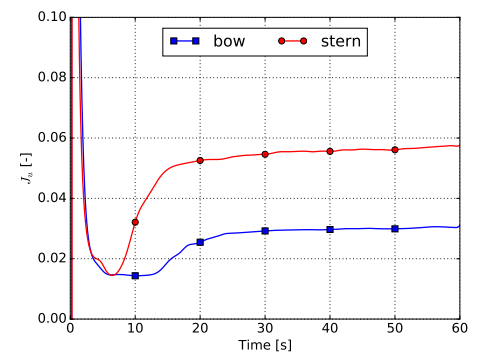

(a) dual-step- $m_{1}$

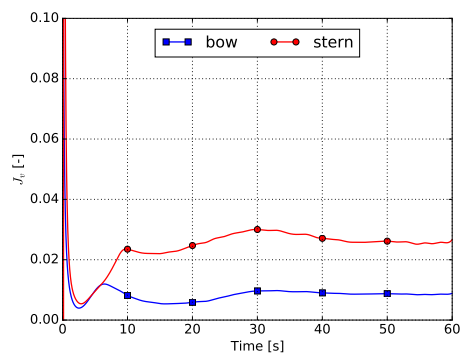

(b) right-step- $m_{1}$

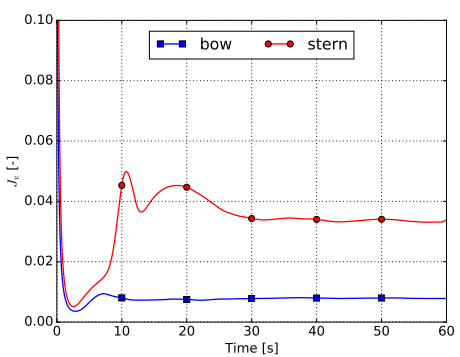

(c) ccw-stairs- $m_{1}$

Figure 18. Pseudo longitudinal, $J_{u}$, or transversal, $J_{v}$, advance speed ratios, at both thrusters for a three-step mission in the three different motion modes: (a) dual-step- $m_{1}$, (b) right-step- $m_{1}$, and (c) $c$ cw-stairs $-m_{1}$.

Whether or not the conventional thruster models of this study capture the conventional advance speed effect of a normal propeller configuration might not be determinable with the current data. It might also be the case that this conventional model structure succeeds in approximating another physical phenomena. Nevertheless, it can be concluded that both speed-dependent thruster models of this study, i.e., (17) and (20), perform better than the speed-independent thruster model, (19), for the surge missions, which did induce higher vessel speeds compared to the sway and yaw cases. For the latter two motion cases, the simpler bollard thrust model (19) seems to suffice to capture the vessel behaviour. Be noted that, running the sway and yaw models without limitations on their speed-dependent thruster parameters, i.e., $T_{n v}^{90, b / s}, T_{n n v}^{90, b / s} \in[0,-\infty]$, resulted in similar identification results where the speed-dependent thrust losses became assigned to only one thruster. This assignment 
might have been caused due to the fact that both propellers received the same shapes of input signals. Future work could add the no-yaw or no-sway constraints to the cost functions of these sway or yaw modes, which might reduce or avoid this identification effect. Finally, for all motions, the damping forces or moments seem to be capturable with only two parameters: a combination of a linear and a quadratic or cubic term. Consequently, it was demonstrated that a variety of model structures can capture the decoupled hydrodynamic behaviour of the Cogge. Evidently, the selection of a model structure depends on the envisaged requirements of its end user.

Future work could also focus on investigating the coupled equations of motion, where the addition of the roll motion might be beneficial, considering the data of Figure 17. Likewise, the propeller dynamics [39] or the potential thruster-thruster interactions [72] could be investigated. These interactions might be present when the outflow of the steering-grid points towards the stern. Adding a wind sensor and explicitly modelling this disturbance should also offer a better performance than the currently implemented bias terms which were included to capture these effects. It should also be noted that, based on Figure 10, the installed flow straightener seems to have significantly decreased the thrust force of the four-channel thruster. Hence, it might be better to replace this configuration with a rudder in the future. Finally, it should be pointed out that the identification methods used need not be restricted to the suggested model structures or the utilised vessel and its actuators. For example, a similar approach could be applied to the real-size Watertruck ${ }^{+}$vessels, which would eliminate the scale effects [73] which would occur when one extrapolates the presently identified models. It is noteworthy that the small scale factor of eight should help in decreasing these scale effects [74].

\section{Conclusions}

This study detailed the geometry of a scale model of a self-propelled CEMT-I barge which houses a novel actuation system configuration consisting of two fully-rotatable and embedded thrusters. Thereupon, this work derived several model structures for the decoupled equations of motion in surge, sway, and yaw, with a focus on the thruster and damping models. The supplementary materials hold all the outdoor experimental data captured to identify and compare these model structures. Accordingly, this work discussed the onboard sensors which fetched these data sets, and detailed the implemented data post-processing. Furthermore, this work offered two identification methods-one based on the instantaneous force balance, and the other one integrated the differential equations of motion. Both methods were used to identify the derived motion models but they could also be used to determine other manoeuvring models. The identified model structures for the surge motion were compared with an external CFD study and with an empirical approach. Subsequently, the conventional thruster model seems to be a good model structure for the surge motion. Speed-independent thrust models seem to suffice for the yaw and sway motions. Finally, cubic or quadratic models seemed to suit to predict the damping forces or moments for surge, sway, and yaw.

Supplementary Materials: S1: Experimental Data: All the experimental data of this study are available online at: http:/ / www.mdpi.com/2077-1312/8/11/889/s1.

Author Contributions: Conceptualization, G.P., S.V.B., G.Y., T.C., M.R.A., Y.S., and P.S.; methodology, G.P. and P.S.; software, G.P., S.V.B., G.Y., and T.C.; validation, G.P.; formal analysis, G.P.; investigation, G.P., S.V.B., G.Y., T.C., M.R.A., C.C., and R.L.; resources, G.P. and C.C.; data curation, G.P., S.V.B. and G.Y.; writing-original draft preparation, G.P.; writing-review and editing, G.P., M.V., R.L., and P.S.; visualization, G.P., S.V.B., and C.C.; supervision, M.V., R.B., and P.S.; project administration, M.R.A. and P.S.; funding acquisition, G.P. and P.S. All authors have read and agreed to the published version of the manuscript.

Funding: The Flanders Research Foundation (FWO) funds the doctoral scholarship of Gerben Peeters, 1S12517N. The Cogge was funded by the EFRO-Flanders project "Autonoom Varen in de Westhoek."

Conflicts of Interest: The authors declare no conflict of interest. 


\section{Abbreviations}

The following abbreviations are used in this manuscript:

$\begin{array}{ll}\text { CCW } & \text { Counter Clockwise } \\ \text { CFD } & \text { Computational Fluid Dynamics } \\ \text { CW } & \text { Clockwise } \\ \text { DEM } & \text { Differential Equation Method } \\ \text { FBM } & \text { Force Balance Method } \\ \text { GCI } & \text { Grid Convergence Index } \\ \text { GNSS } & \text { Global Navigation Satellite System } \\ \text { I-PC } & \text { Industrial Computer } \\ \text { IMU } & \text { Inertial Measurement Unit } \\ \text { MOOS } & \text { Mission Oriented Operating Suite } \\ \text { PLC } & \text { Programmable Logic Controller } \\ \text { PPS } & \text { Pulse Per Second } \\ \text { RTK } & \text { Real Time Kinematic } \\ \text { SP-CEMT-I } & \text { Self-Propelled CEMT Type I barge } \\ \text { UTC } & \text { Coordinated Universal Time }\end{array}$

\section{Appendix A}

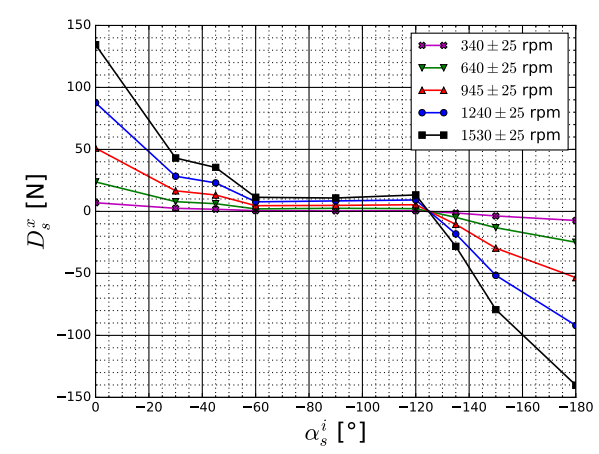

(a)

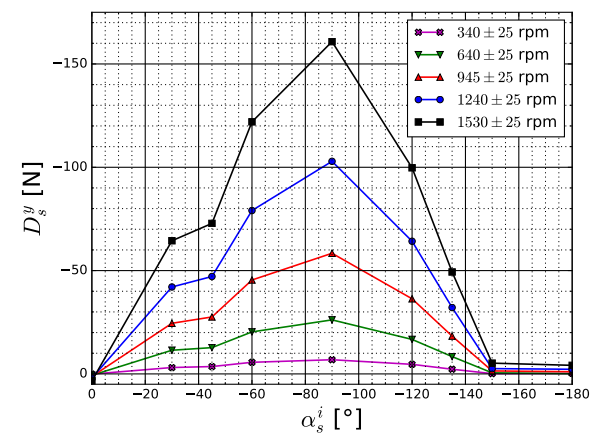

(b)

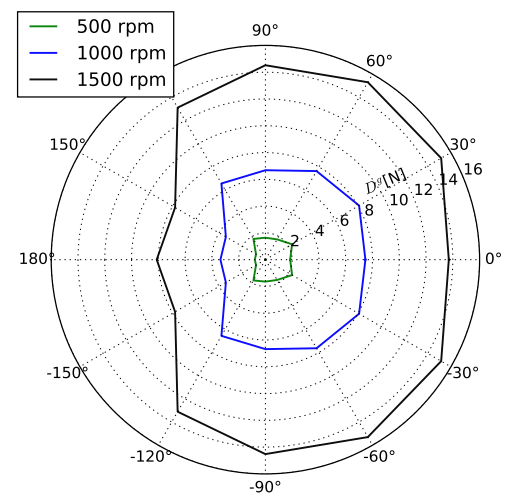

(c)

Figure A1. Experimental bollard pull thruster data: (a) four-channel stern thruster in longitudinal direction, $D_{S}^{x}\left(\alpha_{s}^{i}\right)(\mathbf{b})$ four-channel stern thruster in transversal direction, $D_{S}^{y}\left(\alpha_{s}^{i}\right)$, and (c) steering-grid bow thruster, $D_{b}\left(\alpha_{b}^{i}\right)$. Figures reproduced from [22], with permission from MDPI, 2020. Note that the stern thruster data were fetched with this thruster installed in half a ship hull, whereas the steering-grid was tested as a stand-alone device. Consequently, it may be assumed that the full-internal and external-angle-dependent thrust deductions were captured for the stern thruster, whereas only the, probably dominant, internal angle-dependent deductions were measured for the bow thruster; see [22]. 


\section{Appendix B}

Table A1. Identified surge model coefficients for the DEM ${ }^{u}$.

\begin{tabular}{|c|c|c|c|c|c|c|c|c|c|}
\hline & $\theta_{u}^{a}$ & $\theta_{u}^{b}$ & $\theta_{u}^{c}$ & $\theta_{u}^{d}$ & $\theta_{u}^{e}$ & $\theta_{u}^{f}$ & $\theta_{u}^{g}$ & $\theta_{u}^{h}$ & $\theta_{u}^{i}$ \\
\hline cost & 13.7 & 14.2 & 15.3 & 36.2 & 45.3 & 92.8 & 19.1 & 20.8 & 23.7 \\
\hline$X_{\dot{u}}$ & 26.60 & 35.41 & 75.00 & 75.00 & 25.00 & 62.60 & 43.46 & 74.96 & 56.80 \\
\hline$X_{\text {иии }}$ & 8.30 & 0.00 & 0.00 & 25.85 & 0.00 & 0.00 & 13.14 & 0.00 & 0.00 \\
\hline$X_{u u}$ & 0.00 & 9.01 & 0.00 & 0.00 & 33.08 & 0.00 & 0.00 & 13.45 & 0.00 \\
\hline$X_{u}$ & 14.7 & 11.9 & 18.2 & 16.0 & 3.60 & 39.5 & 18.0 & 15.3 & 24.0 \\
\hline$T_{n n}^{0, b}$ & $6.88 \times 10^{-6}$ & $7.53 \times 10^{-6}$ & $9.03 \times 10^{-6}$ & $6.28 \times 10^{-6}$ & $5.49 \times 10^{-6}$ & $5.46 \times 10^{-6}$ & $5.55 \times 10^{-6}$ & $6.70 \times 10^{-6}$ & $7.29 \times 10^{-6}$ \\
\hline$T_{n n}^{0, s}$ & $2.62 \times 10^{-5}$ & $2.70 \times 10^{-5}$ & $2.93 \times 10^{-5}$ & $1.67 \times 10^{-5}$ & $1.48 \times 10^{-5}$ & $1.69 \times 10^{-5}$ & $2.63 \times 10^{-5}$ & $2.86 \times 10^{-5}$ & $2.95 \times 10^{-5}$ \\
\hline$T_{n v}^{0, b}$ & $-6.84 \times 10^{-3}$ & $-9.22 \times 10^{-3}$ & $-1.41 \times 10^{-2}$ & 0.00 & 0.00 & 0.00 & 0.00 & 0.00 & 0.00 \\
\hline$T_{n v}^{0, s}$ & $-2.67 \times 10^{-2}$ & $-2.86 \times 10^{-2}$ & $-3.23 \times 10^{-2}$ & 0.00 & 0.00 & 0.00 & 0.00 & 0.00 & 0.00 \\
\hline$T_{n n v}^{0, b}$ & 0.00 & 0.00 & 0.00 & 0.00 & 0.00 & 0.00 & $-2.80 \times 10^{-07}$ & $-1.71 \times 10^{-6}$ & $-3.07 \times 10^{-6}$ \\
\hline$T_{n n v}^{0, s}$ & 0.00 & 0.00 & 0.00 & 0.00 & 0.00 & 0.00 & $-1.56 \times 10^{-5}$ & $-1.81 \times 10^{-5}$ & $-1.98 \times 10^{-5}$ \\
\hline
\end{tabular}

Table A2. Identified surge model coefficients for the $\mathrm{FBM}^{B}$.

\begin{tabular}{|c|c|c|c|c|c|c|c|c|c|}
\hline & $\theta_{u}^{a}$ & $\theta_{u}^{b}$ & $\theta_{u}^{c}$ & $\theta_{u}^{d}$ & $\theta_{u}^{e}$ & $\theta_{u}^{f}$ & $\theta_{u}^{g}$ & $\theta_{u}^{h}$ & $\theta_{u}^{i}$ \\
\hline cost & $44,306.5$ & $44,460.3$ & $50,313.2$ & $79,170.6$ & $80,628.9$ & $121,466.91$ & $59,084.0$ & $59,675.91$ & $63,849.7$ \\
\hline$X_{\dot{u}}$ & 25.00 & 25.00 & 25.00 & 25.00 & 25.00 & 25.00 & 25.00 & 25.00 & 25.00 \\
\hline$X_{\text {иии }}$ & 4.42 & 0.00 & 0.00 & 16.60 & 0.00 & 0.00 & 9.36 & 0.00 & 0.00 \\
\hline$X_{\text {ии }}$ & 4.97 & 11.98 & 0.00 & 5.18 & 31.12 & 0.00 & 0.00 & 13.46 & 0.00 \\
\hline$X_{u}$ & 9.43 & 6.52 & 15.35 & 10.10 & 0.00 & 31.07 & 14.22 & 8.92 & 19.04 \\
\hline$T_{n n}^{0, b}$ & $7.00 \times 10^{-6}$ & $7.00 \times 10^{-6}$ & $7.00 \times 10^{-6}$ & $5.18 \times 10^{-6}$ & $5.18 \times 10^{-6}$ & $5.18 \times 10^{-6}$ & $6.27 \times 10^{-6}$ & $6.50 \times 10^{-6}$ & $7.00 \times 10^{-6}$ \\
\hline$T_{n n}^{0, s}$ & $2.41 \times 10^{-5}$ & $2.41 \times 10^{-5}$ & $2.54 \times 10^{-5}$ & $1.47 \times 10^{-5}$ & $1.49 \times 10^{-5}$ & $1.56 \times 10^{-5}$ & $2.37 \times 10^{-5}$ & $2.38 \times 10^{-5}$ & $2.50 \times 10^{-5}$ \\
\hline$T_{n v}^{0, b}$ & $-8.79 \times 10^{-3}$ & $-8.85 \times 10^{-3}$ & $-1.02 \times 10^{-2}$ & 0.00 & 0.00 & 0.00 & 0.00 & 0.00 & 0.00 \\
\hline$T_{n v}^{0, s}$ & $-2.47 \times 10^{-2}$ & $-2.51 \times 10^{-2}$ & $-2.83 \times 10^{-2}$ & 0.00 & 0.00 & 0.00 & 0.00 & 0.00 & 0.00 \\
\hline$T_{n n v}^{0, b}$ & 0.00 & 0.00 & 0.00 & 0.00 & 0.00 & 0.00 & $-2.13 \times 10^{-6}$ & $-2.47 \times 10^{-6}$ & $-3.54 \times 10^{-6}$ \\
\hline$T_{n n v}^{0, s}$ & 0.00 & 0.00 & 0.00 & 0.00 & 0.00 & 0.00 & $-1.47 \times 10^{-5}$ & $-1.51 \times 10^{-5}$ & $-1.66 \times 10^{-5}$ \\
\hline
\end{tabular}

Table A3. Identified surge model coefficients for the $\mathrm{FBM}^{U}$.

\begin{tabular}{|c|c|c|c|c|c|c|c|c|c|}
\hline & $\theta_{u}^{a}$ & $\theta_{u}^{b}$ & $\theta_{u}^{c}$ & $\theta_{u}^{d}$ & $\theta_{u}^{e}$ & $\theta_{u}^{f}$ & $\theta_{u}^{g}$ & $\theta_{u}^{h}$ & $\theta_{u}^{i}$ \\
\hline cost & $43,361.1$ & $43,361.1$ & $44,946.8$ & $144,849.2$ & $85,623.0$ & $228,755.0$ & $59,084.0$ & $450,793.1$ & $63,572.6$ \\
\hline$X_{\dot{u}}$ & 25.00 & 25.00 & 25.00 & 35.45 & 29.47 & 35.42 & 25.0 & 35.40 & 25.00 \\
\hline$X_{\text {иии }}$ & 0.00 & 0.00 & 0.00 & 12.58 & 0.00 & 0.00 & 9.36 & 0.00 & 0.00 \\
\hline$X_{\text {ии }}$ & 8.10 & 8.10 & 0.00 & 13.38 & 26.78 & 0.00 & 0.00 & 7.00 & 0.00 \\
\hline$X_{u}$ & 8.63 & 8.63 & 13.78 & 15.68 & 5.64 & 18.25 & 14.22 & 7.00 & 18.85 \\
\hline$T_{n n}^{0, b}$ & $7.82 \times 10^{-6}$ & $7.82 \times 10^{-6}$ & $8.48 \times 10^{-6}$ & $7.01 \times 10^{-6}$ & $5.32 \times 10^{-6}$ & $6.36 \times 10^{-6}$ & $6.27 \times 10^{-6}$ & $8.96 \times 10^{-6}$ & $7.33 \times 10^{-6}$ \\
\hline$T_{n n}^{0, s}$ & $2.42 \times 10^{-5}$ & $2.42 \times 10^{-5}$ & $2.47 \times 10^{-5}$ & $1.82 \times 10^{-5}$ & $1.55 \times 10^{-5}$ & $1.12 \times 10^{-5}$ & $2.37 \times 10^{-5}$ & $5.64 \times 10^{-5}$ & $2.48 \times 10^{-5}$ \\
\hline$T_{n v}^{0, b}$ & $-1.17 \times 10^{-2}$ & $-1.17 \times 10^{-2}$ & $-1.46 \times 10^{-2}$ & 0.00 & 0.00 & 0.00 & 0.00 & 0.00 & 0.00 \\
\hline$T_{n v}^{0, s}$ & $-2.55 \times 10^{-2}$ & $-2.55 \times 10^{-2}$ & $-2.73 \times 10^{-2}$ & 0.00 & 0.00 & 0.00 & 0.00 & 0.00 & 0.00 \\
\hline$T_{n n v}^{0, b}$ & 0.00 & 0.00 & 0.00 & 0.00 & 0.00 & 0.00 & $-2.13 \times 10^{-6}$ & $-7.49 \times 10^{-6}$ & $-3.88 \times 10^{-6}$ \\
\hline$T_{n n v}^{0, s}$ & 0.00 & 0.00 & 0.00 & 0.00 & 0.00 & 0.00 & $-1.47 \times 10^{-5}$ & $-6.65 \times 10^{-5}$ & $-1.63 \times 10^{-5}$ \\
\hline
\end{tabular}

\section{References}

1. Watertruck+. Watertruck+: The Future of Inland Navigation. Available online: http://www.watertruckplus. eu/ (accessed on 19 October 2020).

2. ECMT. Resolution 92/2 on New Classification of Inland Waterways; Technical Report Infrastructures; European Conference Of Ministers Of Transport: Athens, Greece, 1992.

3. Peeters, G.; Kotzé, M.; Afzal, M.R.; Catoor, T.; Van Baelen, S.; Geenen, P.; Vanierschot, M.; Boonen, R.; Slaets, P. An unmanned inland cargo vessel: Design, build, and experiments. Ocean. Eng. 2020, 201, 17. [CrossRef]

4. Peeters, G.; Yayla, G.; Catoor, T.; Van Baelen, S.; Afzal, M.R.; Christofakis, C.; Storms, S.; Boonen, R.; Slaets, P. An Inland Shore Control Centre for Monitoring or Controlling Unmanned Inland Cargo Vessels. J. Mar. Sci. Eng. 2020, 8, 758. [CrossRef]

5. Zheng, H.; Negenborn, R.R.; Lodewijks, G. Trajectory tracking of autonomous vessels using model predictive control. In Proceedings of the 19th World Congress, The International Federation of Automatic Control, Cape Town, South Africa, 24-29 August 2014; pp. 8812-8818.

6. Eriksen, B.H.; Breivik, M.; Wilthil, E.F.; Flåten, A.L.; Brekke, E.F. The branching-course model predictive control algorithm for maritime collision avoidance. J. Field Robot. 2019, 36, 1222-1249. [CrossRef]

7. Kufoalor, D.K.M.; Johansen, T.A.; Brekke, E.F.; Hepsø, A.; Trnka, K. Autonomous maritime collision avoidance: Field verification of autonomous surface vehicle behavior in challenging scenarios. J. Field Robot. 2019, 37, 387-403. [CrossRef] 
8. Kotzé, M.; Junaid, A.B.; Afzal, M.R.; Peeters, G.; Slaets, P. Use of Uncertainty and Zones for Vessel and Operation in Inland and Waterways. In Journal of Physics: Conference Series, Proceedings of the International Maritime and Port Technology and Development Conference and International Conference on Maritime Autonomous Surface Ships Trondheim, Norway, 13-14 November 2019; IOP Publishing: Bristol, UK, 2019. [CrossRef]

9. Nomoto, K.; Taguchi, T.; Honda, K.; Hirano, S. On the Steering Quality of Ships. Int. Shipbuild. Prog. ISP 1957, 4, 354-370. [CrossRef]

10. Van Amerongen, J. Adaptive steering of ships-A model reference approach. Automatica 1984, 20, 3-14. [CrossRef]

11. Abkowitz, M. Lectures on Ship Hydrodynamics-Steering and Maneuverability; Technical Report; Hydro- and Aerodynamic's Laboratory: Lyngby, Denmark, 1964.

12. Ankudinov, V.; Kaplan, P.; Jacobsen, B.K. Assessment and principal structure of the modular mathematical model for ship maneuverability prediction and real-time maneuvering simulations. In Proceedings of the International Conference on Marine Simulation and Ship Manoeuvrability, St. John's, NL, Canada, 26 September-2 October 1993.

13. Zhang, W.L.Z. Modeling of Ship Maneuvering Motion Using Neural Networks. J. Mar. Sci. Appl. 2016, 15, 426-432.

14. Ogawa, A.; Kasai, H. On the mathematical model of manoeuvring motion of ships. Int. Shipbuild. Prog. 1978, 25, 306-319. [CrossRef]

15. Fossen, T.I. Nonlinear Modeling and Control of Underwater Vehicles. Ph.D. Thesis, Norwegian Institute of Technology, NTNU, Trondheim, Norway, 1991.

16. Fossen, T.I. Guidance and Control of Ocean Vehicles; John Wiley \& Sons. Ltd.: Hoboken, NJ, USA, 1994.

17. Fossen, T.I. Handbook of Marine Craft Hydrodynamics and Motion Control; John Wiley \& Sons, Ltd: Chichester, UK, 2011.

18. Liu, J.; Hekkenberg, R.; Quadvlieg, F.; Hopman, H.; Zhao, B. An integrated empirical manoeuvring model for inland vessels. Ocean. Eng. 2017, 137, 287-308. [CrossRef]

19. Liu, J.; Hekkenberg, R.; Rotteveel, E.; Hopman, H. Literature review on evaluation and prediction methods of inland vessel manoeuvrability. Ocean. Eng. 2015, 106, 458-471. [CrossRef]

20. Peeters, G.; Eggers, A.; Boonen, R.; Slaets, P.; Vanierschot, M. Surge Resistance Identification of Inland Vessels by CFD. In Proceedings of the 2018 OCEANS-MTS/IEEE Kobe Techno-Oceans (OTO), Kobe, Japan, 28-31 May 2018. [CrossRef]

21. Kristensen, H.O.; Lützen, M. Prediction of Resistance and Propulsion Power of Ships; Project No. 2010-56, Emissionsbeslutningsstøttesystem Work Package 2, Report No. 04; Technical University of Denmark: Lyngby, Denmark, 2013.

22. Peeters, G.; Afzal, M.R.; Vanierschot, M.; Boonen, R.; Slaets, P. Model Structures and Identification for Fully Embedded Thrusters: 360-Degrees-Steerable Steering-Grid and Four-Channel Thrusters. J. Mar. Sci. Eng. 2020, 220, 27. [CrossRef]

23. Newman, P.M. MOOS-Mission Oriented Operating Suite; Software, Department of Ocean Engineering Massachusetts Institute of Technology: Cambridge, MA, USA, 2002.

24. Septentrio. AsteRx-U User Manual; Technical Report; Septenrio: Leuven, Belgium, 2017.

25. SBG Sytems. Ekinox Surface Series Tactical Grade MEMS Inertial Sensors Hardware Manual; Technical Report; SBG Systems: Carrières-sur-Seine, Françe, 2018.

26. FLEPOS. The Flemish Positioning Service (Flepos), Flemish Government, Brussels. 2020. Available online: https: / flepos.vlaanderen.be (accessed on 19 October 2020).

27. Yayla, G.; Van Baelen, S.; Peeters, G.; Afzal, M.R.; Catoor, T.; Singh, Y.; Slaets, P. Accuracy Benchmark of Galileo and EGNOS for Inland Waterways. In Proceedings of the International Ship Control Systems Symposium (iSCSS), Delft, The Netherlands, 6-8 October 2020.

28. Lamb, H. Hydrodynamics; C.J. Clay and Sons, Cambrigde University Press: Cambrigde, UK; Victoria University: Manchester, UK, 1895.

29. Newman, J.N. Marine Hydrodynamics, 40th Anniversary Edition ed.; The MIT Press: Cambridge, MA, USA, 2018.

30. WAMIT. The State of the Art in Wave Interaction Analysis. Software, 2020. Available online: https: / / wamit.com/index.htm (accessed on 19 October 2020). 
31. Imlay, F.H. The Complete Expressions for "Added Mass" of a Rigid Body Moving in an Ideal Fluid; Research and Development Report 1528; Department of the Navy David Taylor Model Basin, Hydromechanics Laboratory, Department of the Navy, David Taylor Model Basin: Bethesda, MD, USA, 1961.

32. Zhou, Z.; Yan, S.; Feng, W. Manoeuvring prediction of multiple-purpose cargo ships. Ship Eng. 1983, 6, 21-36. (In Chinese)

33. Clarke, D.; Gedling, P.; Hine, G. Application of manoeuvring criteria in hull design using linear theory. Trans. R. Inst. Nav. Archit. 1983, 125, 45-68.

34. Ross, A. Nonlinear Manoeuvring Models for Ships: A Lagrangian Approach. Ph.D. Thesis, Norwegian University of Science and Technology, NTNU, Department of Engineering Cybernetics, Trondheim, Norway, 2008.

35. Faltinsen, O. Sea Loads on Ships and Offshore Structures; Cambridge University Press: Cambridge, UK, 1990.

36. Fedyaevsky, K.K.; Sobolev, G.V. Control and Stability in Ship Design; State Union Shipbuilding Industry Publishing House: Leningrad, Russia, 1964.

37. Beveridge, J.L. Design and Performance of Bow Thrusters; Technical Report; Naval Ship Research and Development Center Bethesda: Washinton DC, USA, 1971.

38. Baniela, S.I. The performance of a tunnel bow thruster with slow speed ahead: A revisited issue. J. Navig. 2009, 62, 631-642. [CrossRef]

39. Palmer, A.; Hearn, G.E.; Stevenson, P. Modelling Tunnel Thrusters for Autonomous Underwater Vehicles. IFAC Proc. Vol. 2008, 41, 91-96. [CrossRef]

40. Desai-Patil, V.; Ayare, A.; Mahajan, B.; Bade, S. A Review of Azimuth Thruster. Int. J. Mech. Eng. 2015, 2, 38-41. [CrossRef]

41. Dang, J.; Laheij, H. Dynamic Positioning Conference; Technical Report June; Wärtsilä Propulsion Netherlands BV (WPNL): Drunen, The Netherlands, 2004.

42. Triantafyllou, M.S. Steady and Unsteady Dynamics of an Azimuthing Podded Propulsor Related to Vehicle Maneuvering. Ph.D. Dissertation, Department of Ocean Engineering, MIT, Cambridge, MA, USA, 2004.

43. Blanke, M. Ship Propulsion Losses Related to Automatic Steering and Prime Mover Control. PhD Thesis, Technical University of Denmark, Lyngby, Denmark, 1981.

44. Gong, J.; Guo, C.Y.; Wang, C.; Wu, T.C.; Song, K.W. Analysis of waterjet-hull interaction and its impact on the propulsion performance of a four-waterjet-propelled ship. Ocean. Eng. 2019, 180, 211-222. [CrossRef]

45. Altosole, M.; Benvenuto, G.; Figari, M.; Campora, U. Dimensionless Numerical Approaches for the Performance Prediction of Marine Waterjet Propulsion Units. Int. J. Rotating Mach. 2012. [CrossRef]

46. Jiao, W.; Cheng, L.; Zhang, D.; Zhang, B.; Su, Y.; Wang, C. Optimal Design of Inlet Passage for Waterjet Propulsion System Based on Flow and Geometric Parameters. Adv. Mater. Sci. Eng. 2019, 2019, 1-21. [CrossRef]

47. Hou, X.; Guo, S.; Shi, L.; Xing, H.; Liu, Y.; Liu, H.; Hu, Y.; Xia, D.; Li, Z. Hydrodynamic Analysis-Based Modeling and Experimental Verification of a New Water-Jet Thruster for an Amphibious Spherical Robot. Sensors 2019, 19, 259. [CrossRef]

48. Herrero, E.R.; Gonzalez, F.J.V. Two-step identification of non-linear manoeuvring models of marine vessels. Ocean. Eng. 2012, 53, 72-82. [CrossRef]

49. International Towing Tank Conference. Full Scale Manoeuvring Trials_ITTC; Technical Report; International Towing Tank Conference: Zürich, Switzerland, 2017.

50. Peeters, G.; Yayla, G.; Afzal, M.R.; Van Baelen, S.; Catoor, T.; Vanierschot, M.; Boonen1, R.; Slaets, P. Additional Test Manoeuvres for Autonomous Inland Vessels. In Proceedings of the Third International Conference on Maritime Autonomous Surface Ship, ICMASS, Ulsan, Korea, 11-12 November 2020; IOP Conference Series: Material Science and Engineering (MSE): Ulsan, Korea, 2020.

51. The Pandas Development Team. Pandas-Dev/Pandas: Pandas; Version 1.0.3.; The Pandas Development Team: Softwarey, Zenodo, 2020. [CrossRef]

52. Van Rossum, G.; Drake, F.L. Python 3 Reference Manual; CreateSpace: Scotts Valley, CA, USA, 2009.

53. Mohamed Saad IBN SEDDIK. GPS MOOS-Driver (iGPS). Software on Github; The MIT License (MIT), Copyright (c) IBN SEDDIK Mohamed Saad: Cambridge, MA, USA, 2015

54. Harris, C.R.; Millman, K.J.; van der Walt, S.J.; Gommers, R.; Virtanen, P.; Cournapeau, D.; Wieser, E.; Taylor, J.; Berg, S.; Smith, N.J.; et al. Array programming with NumPy. Nature 2020, 585, 357-362. [CrossRef] 
55. Virtanen, P.; Gommers, R.; Oliphant, T.E.; Haberland, M.; Reddy, T.; Cournapeau, D.; Burovski, E.; Peterson, P.; Weckesser, W.; Bright, J.; et al. SciPy 1.0: Fundamental Algorithms for Scientific Computing in Python. Nat. Methods 2020, 17, 261-272. [CrossRef]

56. Liu, Z.; Zhang, Y.; Yu, X.; Yuan, C. Unmanned surface vehicles: An overview of developments and challenges. Annu. Rev. Control 2016, 41,71-93. [CrossRef]

57. Petrich, J. Improved Guidance, Navigation, and Control for Autonomous Underwater Vehicles: Theory and Experiment. Ph.D. Thesis, Virginia Polytechnic Institute and State University, Blacksburg, VA, USA, 2009.

58. Branch, M.A.; Coleman, T.F.; Li, Y. A Subspace, Interior, and Conjugate Gradient Method for Large-Scale Bound-Constrained Minimization Problems. SIAM J. Sci. Comput. 1999, 20, 1-23. [CrossRef]

59. Hindmarsh, A. ODEPACK, a Systematized Collection of ODE Solvers. Sci. Comput. 1983, 1, 55-64.

60. Pillonetto, G.; DiNuzzo, F.; Chen, T.; De Nicolao, G.; Ljung, L. Kernel methods in system identification, machine learning and function estimation: A survey. Automatica 2014, 50, 657-682. [CrossRef]

61. Avila, J.P.; Donha, D.C.; Adamowski, J.C. Experimental model identification of open-frame underwater vehicles. Ocean. Eng. 2013, 60, 81-94. [CrossRef]

62. Sonnenburg, C.R. Modeling, Identification, and Control of an Unmanned Surface Vehicle. Ph.D. Thesis, Virginia Polytechnic Institute and State University, Blacksburg, VA, USA, 2012.

63. Sonnenburg, C.R.; Woolsey, C.A. Modeling, Identification, and Control of an Unmanned Surface Vehicle. J. Field Robot. 2013, 30, 371-398. [CrossRef]

64. Weller, H.G.; Tabor, G. A tensorial approach to computational continuum mechanics using object-oriented techniques. Comput. Phys. 1998, 12, 620-631. [CrossRef]

65. Hirt, C.W.; Nichols, B.D. Volume of fluid (VOF) method for the dynamics of free boundaries. J. Comput. Phys. 1981, 39, 201-225. [CrossRef]

66. Menter, F.R. Two-Equation Eddy-Viscosity Turbulence Models for Engineering Applications. AIAA J. 1994, 32, 1598-1605. [CrossRef]

67. SIMMAN 2014. Workshop on Verification and Validation of Ship Manoeuvring Simulation Models; Experimental Towing Tank Data kvlcc2 Tanker; SIMMAN: Lyngby, Denmark, 2014.

68. Roache, P.J. Perspective: A Method for Uniform Reporting of Grid Refinement Studies. J. Fluids Eng. 1994, 116, 405-413. [CrossRef]

69. Morrall, A. 1957 ITTC Model Ship Correlation Line Values of Frictional Resistance Coefficient; Npl Ship Report 142; ITTC: Zürich Switzerland, 1957.

70. Harvald, S.A. Resistance and Propulsion of Ships; Wiley: Hoboken, NJ, USA, 1983.

71. Motora, S. On the measurement of added mass and added moments of intertia for ship motions. J. Soc. Nav. Archit. Jpn. 1959, 105, 83-89. (In Japanese) [CrossRef]

72. Delefortrie, G.; Tello Ruiz, M.; Vantorre, M. Manoeuvring model of an estuary container vessel with two interacting Z-drives. J. Mar. Sci. Technol. 2018, 23, 739-753. [CrossRef]

73. Dogrul, A.; Song, S.; Demirel, Y.K. Scale effect on ship resistance components and form factor. Ocean. Eng. 2020, 209, 107428. [CrossRef]

74. Guo, C.Y.; Zhong, X.H.; Zhao, D.G.; Wang, C.; Lin, J.F.; Song, K.W. Propulsion performance of large-scale ship model in real sea environment. Ocean. Eng. 2020, 210, 107440. [CrossRef]

Publisher's Note: MDPI stays neutral with regard to jurisdictional claims in published maps and institutional affiliations.

(C) 2020 by the authors. Licensee MDPI, Basel, Switzerland. This article is an open access article distributed under the terms and conditions of the Creative Commons Attribution (CC BY) license (http:/ / creativecommons.org/licenses/by/4.0/). 